\title{
Subchondral bone osteoclasts induce sensory innervation and osteoarthritis pain
}

\author{
Shouan Zhu, ${ }^{1,2}$ Jianxi Zhu, ${ }^{1,3}$ Gehua Zhen, ${ }^{1}$ Yihe Hu, ${ }^{3}$ Senbo An,,${ }^{1,3}$ Yusheng Li, ${ }^{1,3}$ Qin Zheng, ${ }^{4}$ Zhiyong Chen, ${ }^{5}$ Ya Yang, ${ }^{5}$ Mei Wan, ${ }^{1}$ \\ Richard Leroy Skolasky, ${ }^{1}$ Yong Cao,, Tianding Wu, ${ }^{1}$ Bo Gao, ${ }^{1}$ Mi Yang, ${ }^{1}$ Manman Gao, Julia Kuliwaba, ${ }^{6}$ Shuangfei Ni, Lei Wang, ${ }^{1}$ \\ Chuanlong Wu, ${ }^{1}$ David Findlay, ${ }^{6}$ Holger K. Eltzschig, ${ }^{7}$ Hong Wei Ouyang, ${ }^{2,8}$ Janet Crane, ${ }^{1}$ Feng-Quan Zhou, ${ }^{1}$ Yun Guan, ${ }^{5}$ \\ Xinzhong Dong, ${ }^{4}$, and $\mathrm{Xu} \mathrm{Cao}^{1}$ \\ 'Department of Orthopaedic Surgery, Johns Hopkins University School of Medicine, Baltimore, Maryland, USA. Dr. Li Dak Sum and Yip Yio Chin Center for Stem Cells and Regenerative Medicine, School \\ of Medicine, Zhejiang University, Hangzhou, China. ${ }^{3}$ Department of Orthopaedic Surgery, Xiangya Hospital, Central South University, Changsha, China. ${ }^{4}$ Department of Neuroscience, Neurosurgery, \\ and Dermatology, Center of Sensory Biology, Johns Hopkins University School of Medicine, Howard Hughes Medical Institute, Baltimore, Maryland, USA. ${ }^{5}$ Department of Anesthesiology and Critical \\ Care Medicine, Johns Hopkins University School of Medicine, Baltimore, Maryland, USA. ${ }^{6}$ Department of Orthopaedics and Trauma, Royal Adelaide Hospital, University of Adelaide, Adelaide, Australia. \\ ${ }^{7}$ Department of Anesthesiology, University of Texas Health Science Center at Houston, McCovern Medical School, Houston, Texas, USA. ${ }^{8}$ ZjU-UoE Joint Institute, School of Medicine, Zhejiang University, \\ Hangzhou, China
}

\begin{abstract}
Joint pain is the defining symptom of osteoarthritis $(\mathrm{OA})$ but its origin and mechanisms remain unclear. Here, we investigated an unprecedented role of osteoclast-initiated subchondral bone remodeling in sensory innervation for OA pain. We show that osteoclasts secrete netrin-1 to induce sensory nerve axonal growth in subchondral bone. Reduction of osteoclast formation by knockout of receptor activator of nuclear factor kappa-B ligand (Rankl) in osteocytes inhibited the growth of sensory nerves into subchondral bone, dorsal root ganglion neuron hyperexcitability, and behavioral measures of pain hypersensitivity in OA mice. Moreover, we demonstrated a possible role for netrin-1 secreted by osteoclasts during aberrant subchondral bone remodeling in inducing sensory innervation and OA pain through its receptor DCC (deleted in colorectal cancer). Importantly, knockout of Netrin1 in tartrate-resistant acid phosphatase-positive (TRAP-positive) osteoclasts or knockdown of Dcc reduces $\mathrm{OA}$ pain behavior. In particular, inhibition of osteoclast activity by alendronate modifies aberrant subchondral bone remodeling and reduces innervation and pain behavior at the early stage of $\mathrm{OA}$. These results suggest that intervention of the axonal guidance molecules (e.g., netrin-1) derived from aberrant subchondral bone remodeling may have therapeutic potential for OA pain.
\end{abstract}

\section{Introduction}

Osteoarthritis (OA) is a common musculoskeletal disease in adults, and it is estimated that it will affect 78 million people by 2040 (1), leading to disability and reduced quality of life. Joint pain is the defining symptom of OA, and yet there is little understanding of its etiology (2). Currently, OA pain is inadequately controlled by analgesics and nonsteroidal antiinflammatory drugs, with unsustained pain relief and substantial adverse effects (3). More recently, humanized nerve growth factor (NGF) mAb has shown great potential in alleviating pain in patients with severe OA (4). However, side effects including association with rapid progression of OA and osteonecrosis in a clinical trial, and autonomic nervous system toxicity in a preclinical model (5) were detected. These observations suggest that a better understanding of OA pain pathomechanisms is essential to develop disease-modifying therapy for OA pain.

Authorship note: SZ and JZ contributed equally to this work. Conflict of interest: The authors have declared that no conflict of interest exists. License: Copyright 2019, American Society for Clinical Investigation.

Submitted: April 10, 2018; Accepted: December 7, 2018.

Reference information: J Clin Invest. 2019;129(3):1076-1093. https://doi.org/10.1172/JCI121561.
Evidence from both clinical and preclinical studies suggests that continuous nociceptive input from the osteoarthritic joint drives sensitization of both central and peripheral nervous systems $(6,7)$. Central sensitization in the spinal cord and dysregulation of the ascending and descending pathways from the brain through the spinal cord, at least partially explains widespread pain sensitivity in OA patients $(8,9)$. Additionally, modulation of an integrated network among glial cells, neurons, and immune cells in the dorsal root ganglion (DRG) and central nervous system has been shown to correlate with arthritis pain $(10,11)$. On the other hand, locally in osteoarthritic joints, cytokines, chemokines, and inflammatory factors, including tumor necrosis factor (TNF) (12), interleukin-1 (IL-1) $(13,14)$, IL-6 $(15,16)$, IL-17 $(17,18)$, NGF $(19-$ $22)$, and prostaglandin E2 $(23,24)$, can lead to hypersensitivity with exaggerated pain (hyperalgesia) by noxious stimuli or innocuous stimuli that are perceived as painful (allodynia). Peripheral sensitization has been evaluated by behavioral testing in ample preclinical models to indicate OA pain (25). As a base for peripheral sensitization, multiple tissues including the synovium (26), ligament (27), osteochondral junction $(28,29)$, and meniscus (30) in the joint are densely innervated by perivascular sensory and sympathetic nerves. Examination of innervation changes in either animal models of OA or human specimens has reached inconsis- 
tent conclusions, probably because different disease stages were observed. Two studies in the collagenase-induced model reported either a transient (31) or a permanent (32) decrease in synovial innervation, while another study using surgical destabilization of the medial meniscus (DMM) and $P k c \delta$-null models reported increased synovial innervation (26). Neural elements revealed by gold chloride staining were initially reported to decrease in osteoarthritic posterior cruciate ligament (PCL) (33), while another study using immunohistochemistry to detect calcitonin gene-related peptide (CGRP) showed constant nociceptive sensory innervation in osteoarthritic PCL (27). In particular, perivascular sensory and sympathetic nerve fibers have been observed breaching the osteochondral junction in OA $(28,29)$.

Subchondral bone may also be an important source of pain in OA; specifically, subchondral bone marrow edema-like lesions visualized by magnetic resonance imaging (MRI) highly correlated with OA pain $(34,35)$. Zoledronic acid, a drug that inhibits osteoclast activity, was effective in reducing osteoarthritic knee pain and bone marrow edema-like lesion size (36). Analysis of a comprehensive data set from the NIH Osteoarthritis Initiative showed that bisphosphonate users experienced significantly reduced knee pain at 2 and 3 years (37). Increased subchondral bone remodeling occurs during $\mathrm{OA}$ progression (38). We reported previously that aberrant subchondral bone remodeling initiates joint articular cartilage degeneration (39). Specifically, elevated osteoclast activity activates excessive TGF- $\beta 1$ production to recruit mesenchymal stem cells in the marrow, where they undergo aberrant subchondral bone formation. Systemic or local administration of TGF- $\beta 1$-neutralizing antibody (1D11) attenuated OA progression by targeting subchondral bone pathological features (40). The subchondral bone changes in the early stage of OA further suggest a potential pathogenesis of OA pain.

In the mammalian nervous system, wiring of neuronal axons into tissues is directed by specific cues in the extracellular environment, a process called axon guidance (also called axon pathfinding). Guidance cues come in 4 types: netrins, Slits, ephrins, and semaphorins. These signals can be fixed in place or diffusible, and they can attract or repel axons. Neurite outgrowth includes elongation and branching (41), and is required by both attractive and repulsive cues to control, respectively, axon outgrowth and disassembly of adhesive structures together with cytoskeletal dynamics $(42,43)$. Interestingly, researchers using a combination of genetic and biochemical methods have found that axon guidance molecules, such as semaphorins, netrins, and ephrins, are also involved in differentiation and communication between osteoclasts and osteoblasts (44-51) essential for bone formation and skeletal homeostasis. Semaphorin 3A (Sema3A) has also been shown to regulate bone remodeling indirectly by modulating sensory nerve innervation (45). Here, we investigated the role of osteoclast-initiated subchondral bone remodeling in sensory innervation for pain hypersensitivity during OA progression. We found that an increase in osteoclasts in early OA was strongly related to the appearance and persistence of sensory nerves in the subchondral bone, with evidence for a role of osteoclast-derived netrin-1 in mediating OA pain.

\section{Results}

Sensory nerve innervation in subchondral bone correlates with osteoclast activity during $O A$ progression. We first examined the poten- tial role of osteoclasts in sensory innervation in subchondral bone, because we have shown an increase in osteoclasts at the early stage of OA and angiogenesis induced by preosteoclasts (39, 52). Mouse anterior cruciate ligaments (ACLs) were transected to create an ACL transection (ACLT) OA model. The ACLT OA joints were harvested at different time points for immunohistological analysis of subchondral bone. At 2 weeks after surgery, we observed decreased proteoglycan staining (red) and a rough surface in articular cartilage, suggesting cartilage matrix degeneration. At 4 weeks after surgery, there were small cartilage lesions across the tibial articular cartilage with big lesions deep within calcified cartilage at 8 weeks (Figure 1A). Tartrate-resistant acid phosphatase-positive (TRAP-positive) osteoclasts were increased in subchondral bone as early as 1 week after ACLT surgery and were maintained at a high level for 2 weeks (Figure 1, A and B). Osteoclastic bone resorption generated large bone marrow cavities at 8 weeks (Figure $1 \mathrm{~A}$ ). We then examined the neurons that innervate subchondral bone. Both posterior and anterior areas of tibial subchondral bone were imaged and analyzed (Supplemental Figure 2, whole-joint CGRP immunostaining; see supplemental material available online with this article; https://doi.org/10.1172/ JCI121561DS1). Immunostaining of CGRP, a potent vasodilator that causes pain sensitization, showed aberrant distribution of peptidergic nociceptive nerve fibers adjacent to the trabecular bone surface beginning 1 week after surgery. The numbers and density of nerve endings remained increased at 8 weeks after ACLT surgery (Figure 1, A and C). Very few $\mathrm{TRAP}^{+}$osteoclasts and CGRP ${ }^{+}$ sensory nerve endings in subchondral bone were observed in sham groups at corresponding time points (Supplemental Figure 1, A and D). Based on a newly proposed classification of sensory neurons (53), we also stained another 3 markers of nociceptive neurons: NF200, P2X2, and PIEZO2. Interestingly, the density of P2X2 and PIEZO2 was also increased, while NF200 remained constant in subchondral bone marrow of ACLT mice (Supplemental Figure 3 , A and B). Staining for PGP9.5 and $\beta$ tubulin to detect other subsets of neuronal fibers in subchondral bone marrow showed minimal alterations by ACLT surgery (Supplemental Figure 3, A and B). Together, these results suggest that the overall innervation of different subgroups of nociceptive neurons is increased in osteoarthritic subchondral bone. Because it has been shown that cartilage degeneration and subchondral bone destruction seem to develop preferentially at the posterior part of the knee (54), we further analyzed the distribution of $\mathrm{CGRP}^{+}$nerves in the 2 different compartments of subchondral bone. Interestingly, no significant difference was observed between the posterior and anterior compartments (Supplemental Figure 3C).

To evaluate whether sensory nerve innervation in subchondral bone is associated with OA pain, we analyzed DRG neuronal activity in Pirt-GCaMP3 mice. In Pirt-GCaMP3 mice, the entire coding region of the phosphoinositide-interacting regulator of TRP (Pirt) gene (55), which is expressed predominantly in nociceptive neurons, was replaced with a $\mathrm{Ca}^{2+}$ indicator (GCaMP3) in frame with the Pirt promoter so that DRG neurons expressed the genetically encoded $\mathrm{Ca}^{2+}$-sensitive indicator (56). This mouse model allows for the detection of increased peripheral neuronal activity in primary sensory neurons in the DRG. We observed significantly increased numbers of activated DRG neurons in response to mechanical 
A
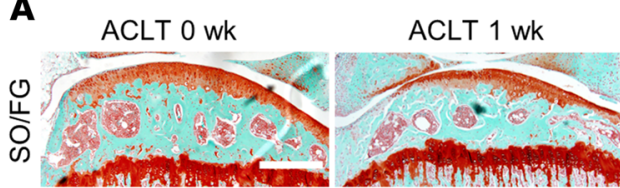

ACLT 2 wks

ACLT 4 wk

ACLT 8 wk
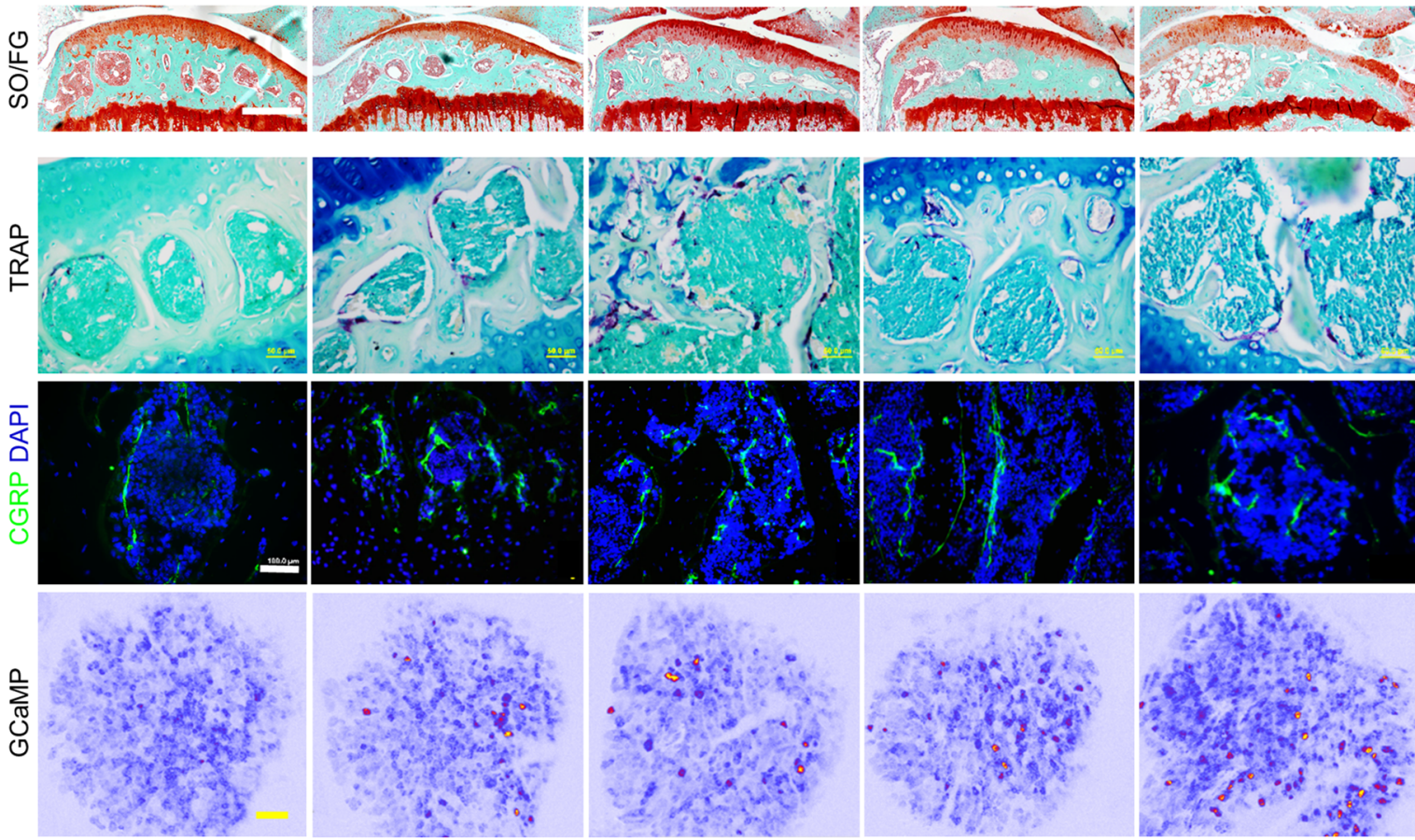

B

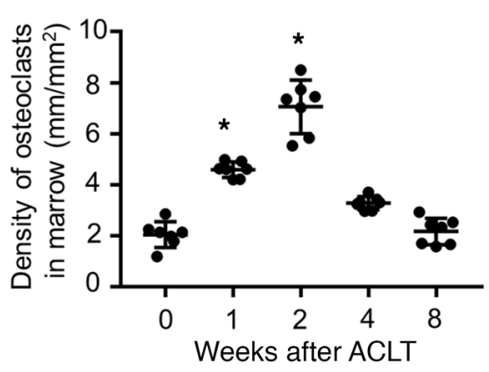

E

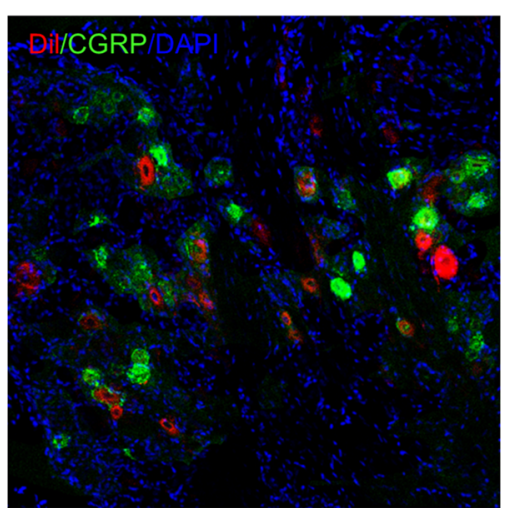

C

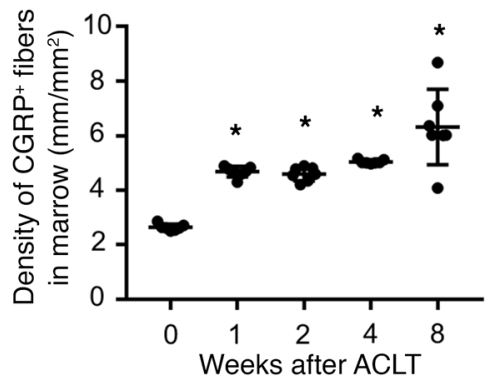

ACLT

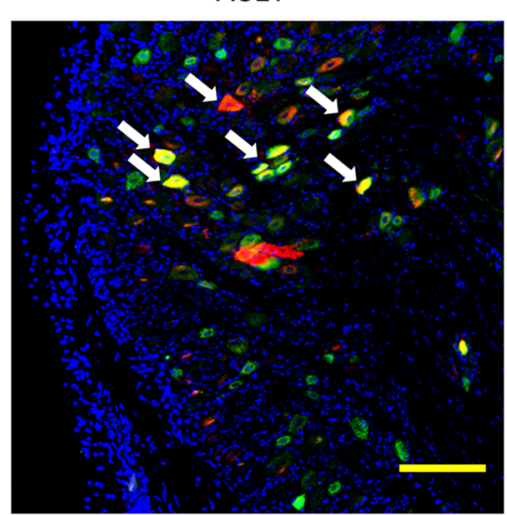

D

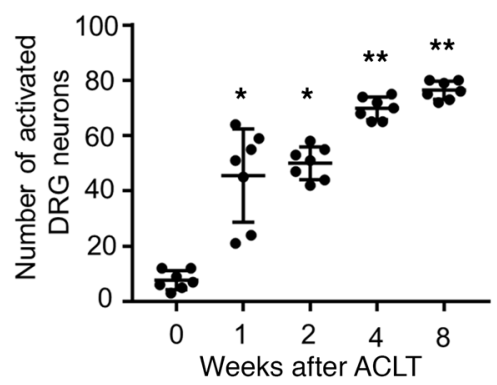

$\mathbf{F}$

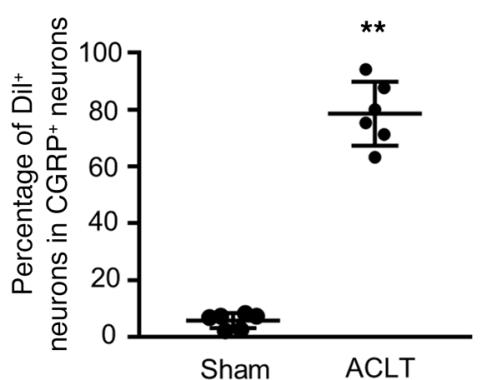

Figure 1. CGRP' sensory nerves in subchondral bone increased along with an increase in osteoclast activity and DRG neuron hypersensitivity during OA progression. (A) Safranin orange/fast green (SO/FC) staining (first row), TRAP staining (second row, magenta), and immunofluorescence analysis of CGRP ${ }^{+}$sensory nerve fibers (third row, green) in mouse tibial subchondral bone after ACLT surgery at different time points. Scale bars: $500 \mu \mathrm{m}$ (first row), $100 \mu \mathrm{m}$ (second row), and $50 \mu \mathrm{m}$ (third row). Excitability (fourth row) of L4 DRG in Pirt-GCaMP3 mice at different time points after surgery. Scale bar: $100 \mu \mathrm{m}$. $n=7$ per time point (see neuronal hyperactivity in Supplemental Videos 1-6). (B and C) Quantitative analysis of density of TRAP ${ }^{+}$and CGRP ${ }^{+}$sensory nerves in subchondral bone marrow. ${ }^{*} P<0.05$ compared with the sham-operated group at the corresponding time points. $n=7$ per time point. (D) Quantification of activated DRG neurons. ${ }^{*} P<0.05$, ${ }^{*} P<0.01$ compared with the sham-operated group at the corresponding time points. $n=7$ per group. (E) Representative photomicrographs of CGRP and Dil double-labeled neurons in L4 DRG. Scale bar: $50 \mu \mathrm{m} . n=6$ per group. (F) Percentage of L4 DRG neurons retrogradely labeled with Dil in all CGRP' neurons 10 weeks after sham or ACLT surgery. ${ }^{* *} P<0.01$ compared with the sham-operated group at the corresponding time points. Statistical significance was determined by multifactorial ANOVA, and all data are shown as means \pm standard deviations. 
A

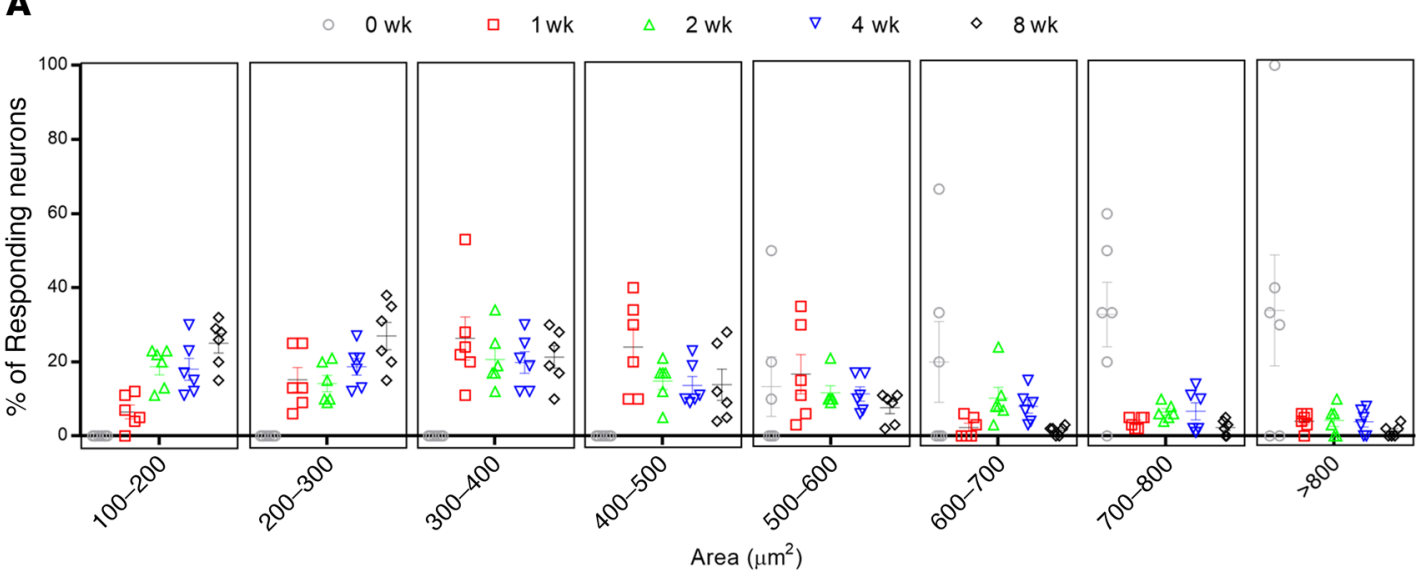

B

Knee pinch-evoked neuron Capsaicin-evoked neuron

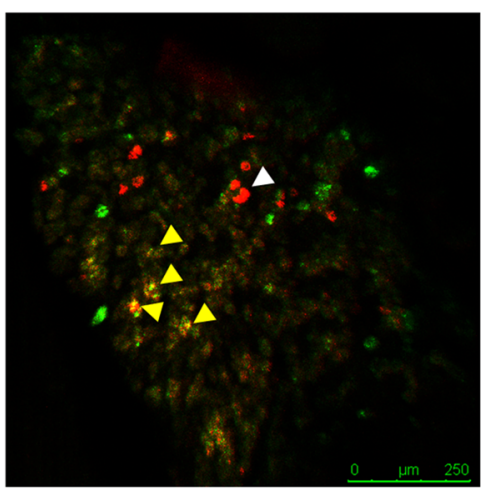

C

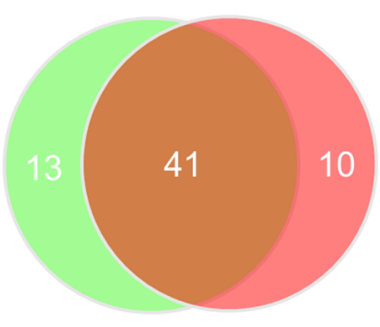

No. of neurons only responding to knee pinch

No. of neurons responding

to knee pinch and capsaicin

No. of neurons only responding to capsaicin
Figure 2. In OA, most DRC neurons responding to knee pinch are nociceptive neurons. (A) Relative frequency distributions of the areas of neurons responding to 20 -g knee pinch in ACLT mice at different time points. Mean \pm standard deviation. (B) Excitability of L4 DRG in Pirt-GCaMP3 mice responding to knee pinch or direct drop of $1 \mu \mathrm{M}$ capsaicin. White arrows indicate neurons responding only to capsaicin; yellow arrows indicate neurons responding to both knee pinch and capsaicin. Scale bar: $250 \mu \mathrm{m}$. (C) Number of DRG neurons responding to knee pinch or capsaicin. force generated by a rodent pincher analgesia meter on the knee at 1 week after ACLT surgery, which had increased to $70 \pm 5$ neurons at 4 weeks and remained steady at 8 weeks (Figure 1 , A and D). In contrast, an average of 5-8 neurons were activated by the same mechanical force in sham-operated mice (Supplemental Figure 1, $\mathrm{B}$ and $\mathrm{C}$ ). Similar neuronal hyperexcitability in a DMM OA mouse model was also recently reported by Miller and colleagues (57).

To validate the increased number of DRG neurons responding to knee pinch through $\mathrm{CGRP}^{+}$sensory innervation in subchondral bone, we conducted a retrograde labeling experiment using 1,1'-dioctadecyl-3,3,3',3'-tetramethylindocarbocyanine perchlorate (Dil) in rats (rats were used instead of mice because of the technical difficulty of injecting dye into subchondral bone in mice). Indeed, the number of $\mathrm{CGRP}^{+}$neurons labeled with Dil in L4-L5 DRG neurons in the ACLT group was significantly greater than that in the sham-surgery group (Figure 1, E and F, and Supplemental Figure 10). The number of IB4 ${ }^{+}$neurons labeled with Dil was not significantly different between the 2 groups (Supplemental Figure 3, D and E). The total number of neurons labeled with Dil in the sham group was $25 \pm 5\left(\sim 5.2 \%\right.$ were $\left.\mathrm{CGRP}^{+}\right)$. The total number of neurons labeled with Dil in the ACLT group was $31 \pm 3$ $\left(\sim 78.3 \%\right.$ were $\left.\mathrm{CGRP}^{+}\right)$(Figure $1, \mathrm{E}$ and $\left.\mathrm{F}\right)$.

To define the specific type of neurons that responded to knee pinch, we assessed the size distribution of the neurons activated by knee pinch during the time course of OA development. Before
ACLT, a few neurons with areas greater than $600 \mu \mathrm{m}^{2}$ were activated by approximately 20-g knee pinch, consistent with the size of non-nociceptive neurons. The number of small- to medium-sized neurons (area $<600 \mu \mathrm{m}^{2}$ ) increased continuously in response to knee pinch after surgery and became the majority of activated neurons at 8 weeks, consistent with the size of $\mathrm{C}$ - and $\mathrm{A} \delta$-fiber neurons, which function primarily as nociceptors (Figure 2A).

To examine whether the increased number of DRG neurons responding to knee pinch were the neuronal population responsible for OA pain, we tested whether they are also capsaicin sensitive. We performed both mechanical force-evoked, as well as capsaicin-evoked in vivo DRG imaging experiments on the same L4 DRG of the same OA mice. With knee pinch, 54 neurons were activated, $76 \%$ of which were also activated by a direct drop of capsaicin $(1 \mu \mathrm{M})$ onto the DRG (Figure 2, B and C). Because capsaicin can activate a subset of primary afferent neurons associated with both pain and thermoreception, some large neurons with greater brightness were activated only by capsaicin (Figure 2B, white arrow).

Together, these findings suggest that an increase in osteoclastmediated bone resorption induces sensory innervation in the subchondral bone and hyperexcitability of DRG neurons. The high correlation between bone remodeling and innervation of nociceptive neurons in subchondral bone suggests that nociceptive neurons could potentially mediate OA pain and be targeted locally. 
A
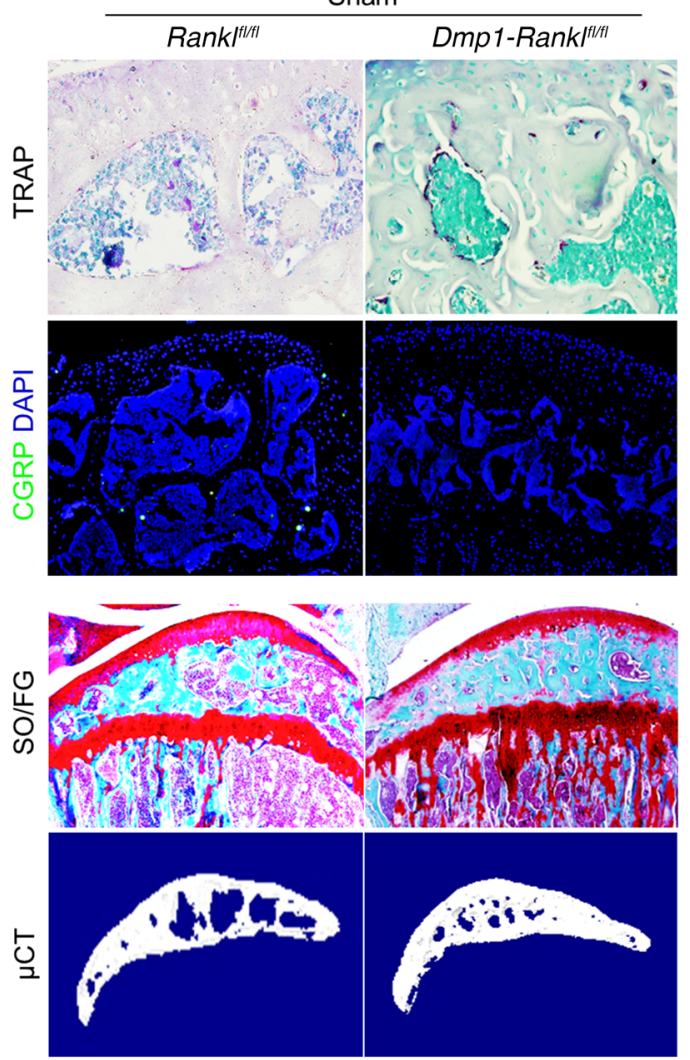

$\mathbf{F}$
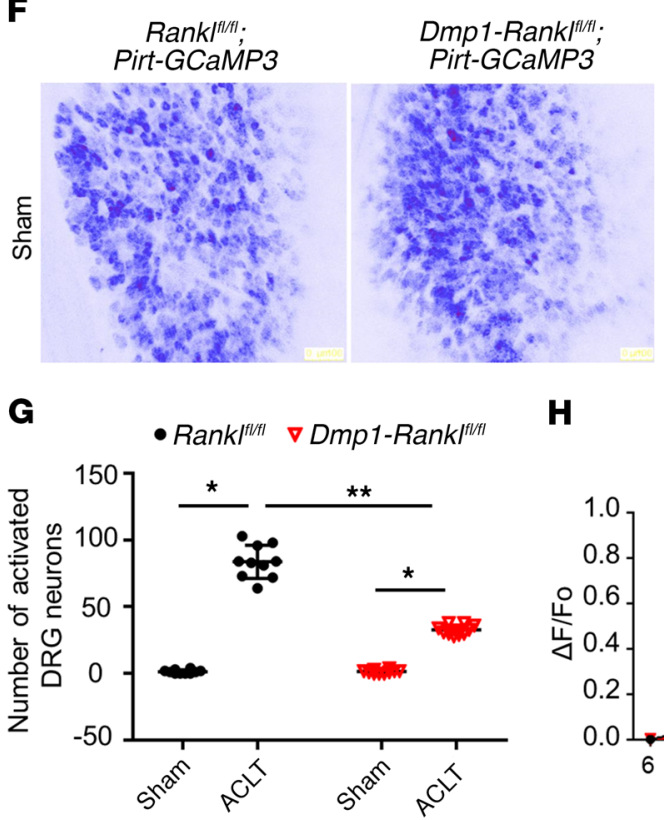

H
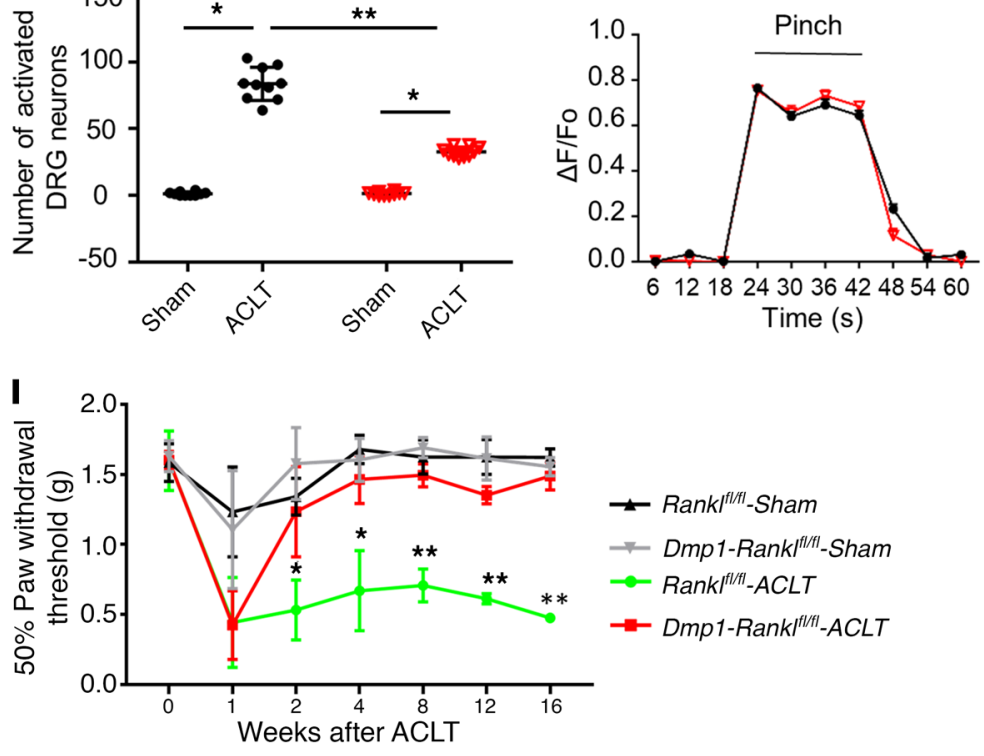

B
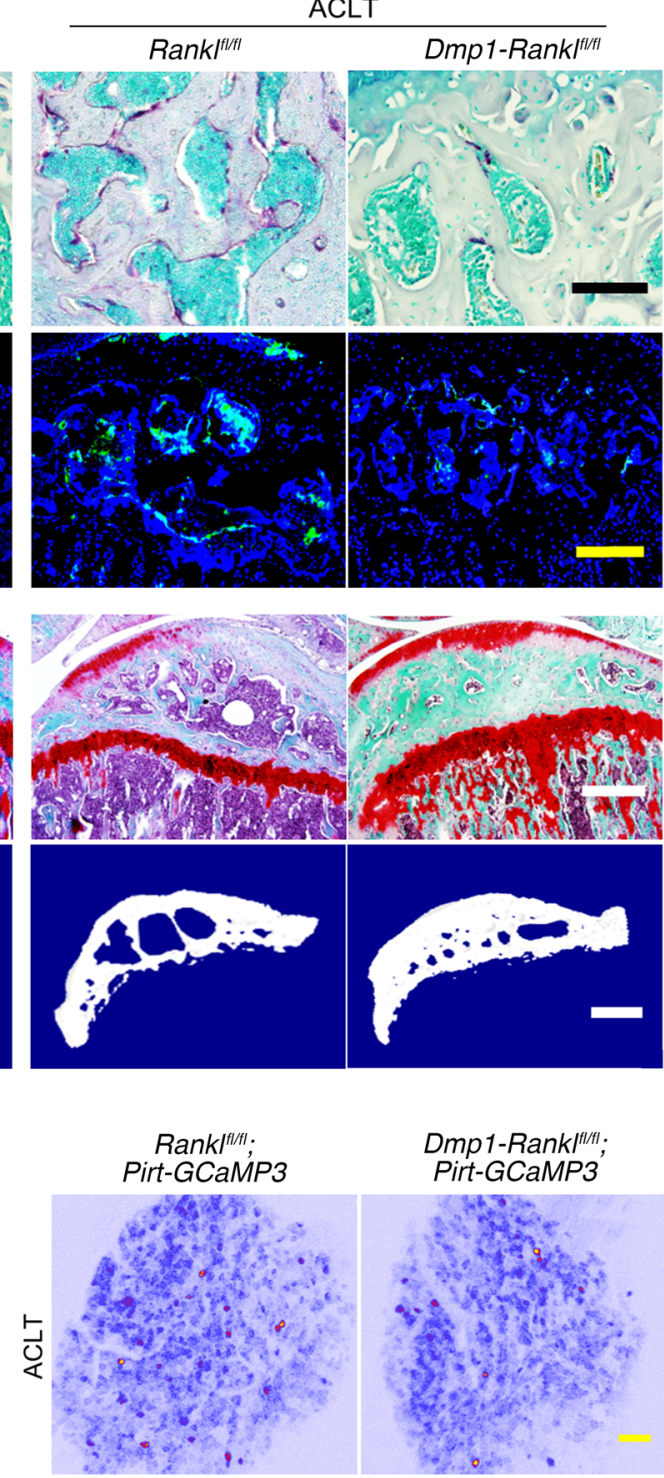

Dmp1-Ranklffifi; Pirt-GCaMP3

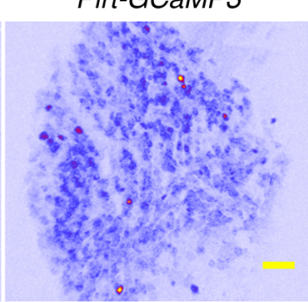

J

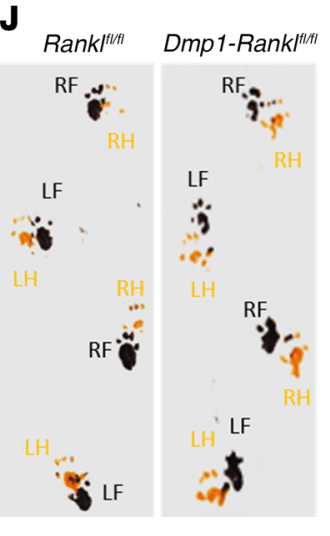

C

D

$\mathbf{K}$
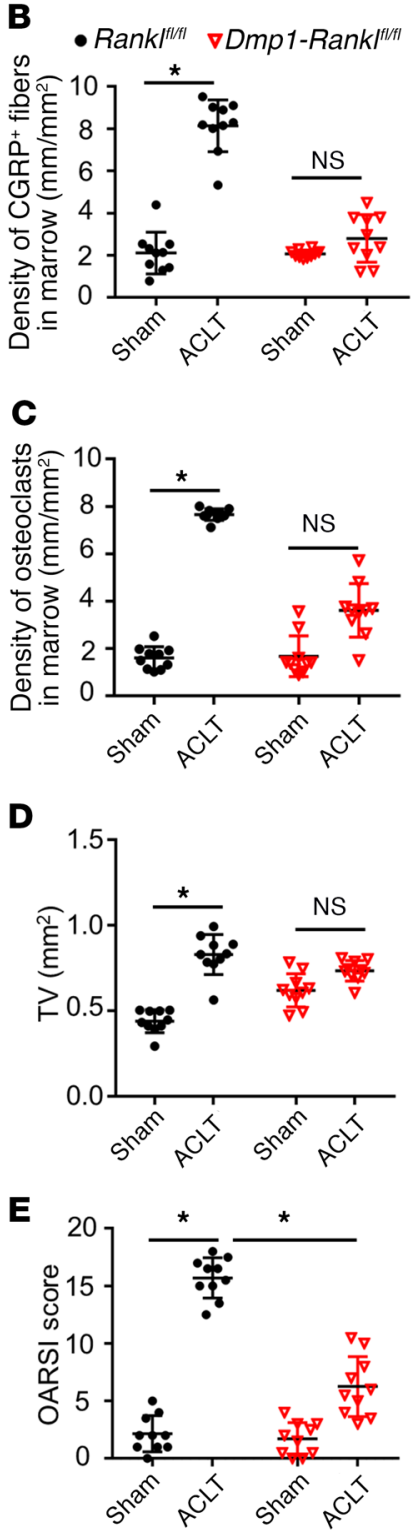

- Rank $\left.\right|^{f / f i l} \quad \nabla$ Dmp1-Rankl $\left.\right|^{[/ f t}$
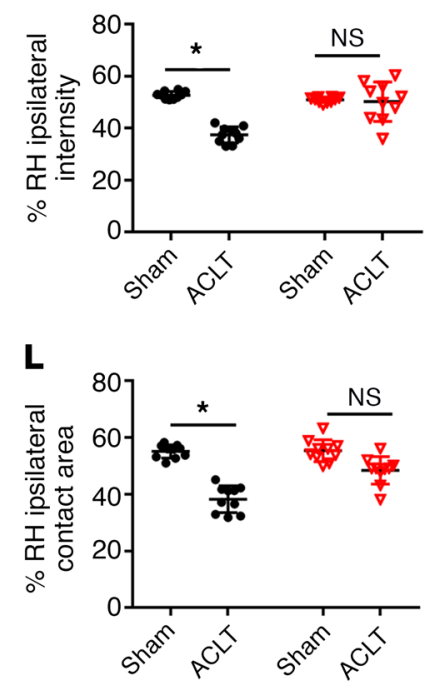
Figure 3. Decreased sprouting of CCRP' sensory nerves in the subchondral bone and pain amelioration in Dmp1-Rank ${ }^{f / f / f}$ mice. (A) TRAP staining (first row, magenta) and immunofluorescence analysis of CGRP' sensory nerve fibers (second row, green) in mouse tibial subchondral bone after ACLT surgery. Scale bars: $100 \mu \mathrm{m}$. Third row: Safranin orange and fast green (SO/FC) staining of articular cartilage in sagittal sections of tibial medial compartment of Rank $k^{f l / f l}$ and Dmp1-Rank $\left.\right|^{f / f f l}$ mice with or without ACLT surgery. Scale bar: $500 \mu \mathrm{m}$. Fourth row: 3-Dimensional $\mu \mathrm{CT}$ image of tibial subchondral bone medial compartment (sagittal view) of Rank $\left.\right|^{f / f f l}$ and Dmp1-Rank $\left.\right|^{f / f f l}$ mice with or without ACLT surgery. Scale bar: $1 \mathrm{~mm} . n=9 /$ group. (B and C) Quantitative analysis of the density of TRAP' osteoclasts and CGRP+ nerve fibers in subchondral bone marrow. ( $\mathbf{D}$ and $\mathbf{E}$ ) Quantitative analysis of total tissue volume (TV) (D) and OARSI scores 8 weeks after surgery (E). $n=9$ /group. (F) In vivo calcium imaging in whole L4 DRG primary sensory neurons after mechanical press to knees of Rank $\left.\right|^{f / f f ;} ;$ Pirt-GCaMP3 and Dmp1-Rank $\left.\right|^{f / f f} ;$ Pirt-GCaMP3 mice. Scale bar: $50 \mu \mathrm{m} . n=10 /$ group. (C) Number of neurons activated by mechanical press. $(\mathbf{H}) \Delta \mathrm{F} / \mathrm{Fo}$ for neurons in a representative $\mathrm{DRG}$ responding to an approximately 20-g paw pinch in Rank $\left.\right|^{\mathrm{fl} / \mathrm{fl}}$ (black) and Dmp1-Rank $\left.\right|^{\mathrm{fl} / \mathrm{fl}}$ (red) mice after ACLT. (I) Paw withdrawal threshold (PWT) was tested at the

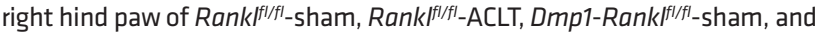

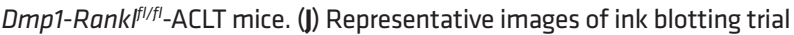
of Rank $\left.\right|^{f l / f l}$ and Dmp1-Rank $f^{f l / f l}$ mice after ACLT surgery on right knees. RH, right hind (orange); LH, left hind (orange); RF, right front (black); LF, left front (black). (K and $\mathbf{L}$ ) Percentage RH ipsilateral intensity (K) and percentage RH ipsilateral contact area (L) were calculated using Image) software. $n=10$ / group. All data are shown as means \pm standard deviations. ${ }^{*} P<0.05$, ${ }^{* *} P<0.01$ by multifactorial ANOVA. NS, no significant difference.

Sprouting of sensory nerves in subchondral bone and $O A$ pain decreased in Dmp1-Rankllf/ll mice. We next tested whether sensory innervation is initiated by osteoclasts and associated with OA pain. Dentin matrix acidic phosphoprotein 1 (Dmp1)-Cre mice were crossbred with receptor activator of nuclear factor kappa-B ligand-floxed (Rankllffl) mice to knock out Rankl in DMP1+ osteocytes. $\mathrm{DMP} 1^{+}$osteocytes are the primary source of Rankl for osteoclast differentiation $(58,59)$. Deficiency of Rankl in osteocytes leads to a decrease in osteoclast number and a severe osteopetrotic phenotype $(58,59)$. TRAP $^{+}$osteoclasts were decreased in the subchondral bone surface in Dmp1-Rank $k^{f / f l}$-ACLT mice relative to Rank $l^{l / f l}$-ACLT controls (Figure 3, A and C). Importantly, the density of $\mathrm{CGRP}^{+}$neurofilaments was markedly decreased in Dmp1-Rank $l^{t / f l}$-ACLT mice (Figure 3, A and B), suggesting that osteoclast activity was associated with $\mathrm{CGRP}^{+}$sensory innervation in the subchondral bone. Moreover, the articular cartilage was protected in Dmp1-Rankllfll-ACLT mice, as indicated by proteoglycan staining (Figure 3A) and significantly lower Osteoarthritis Research Society International (OARSI) scores (51) than those of Rankll/fl-ACLT controls (Figure 3E). The tibial subchondral bone volume in the Rank $l^{[t / f l}$-ACLT mice was $20 \%$ higher than that of sham-surgery controls at 2 months after surgery by microcomputed tomography $(\mu \mathrm{CT})$ analysis. Subchondral bone tissue volume increased slightly (not significant) in Dmp1-Rank $l^{t / f l}$ ACLT mice (Figure 3, A and D). The thickness of the subchondral bone plate was decreased in the Rank $l^{f / f l}$-ACLT mice but remained the same in Dmp1-Rank $l^{f / f l}$-ACLT mice at 2 months after surgery compared with the sham-surgery controls (Supplemental Figure 4A). The trabecular pattern factor was increased in Dmp1-Rank $\|^{t / f_{-}}$ ACLT mice, but not as much as that in Rankll/fl-ACLT mice (Supplemental Figure 4B). Immunostaining showed that the numbers of osterix ${ }^{+}$osteoblast progenitors and $\mathrm{pSMAD} 2 / 3^{+}$cells, which are indicators of increased bone remodeling $(39,60)$, also increased significantly in Rank $l^{t / f l_{-}}$ACLT controls but not in Dmp1-Rank $l^{t / f l_{-}}$ ACLT mice, indicating minimal subchondral bone remodeling in the knockout (KO) mice (Supplemental Figure 4, C-E). Microfil contrast-enhanced angiography also demonstrated abrogation of the increase in subchondral blood vessels in Dmp1-Rank $l^{\text {th/fl }}$ mice relative to Rankll/fl mice after ACLT (Supplemental Figure 4, $\mathrm{F}-\mathrm{H})$. These results suggest that uncoupled bone remodeling was arrested in Dmp1-Rank $l^{f / f l}$-ACLT mice and led to decreased sprouting of sensory nerves.

To examine whether sensory nerves in subchondral bone mediate OA pain, we next crossbred Dmp1-Rank $\left.\right|^{l / f l}$ with PirtGCaMP3 mice. Compared with that in Rankllfli; Pirt-GCaMP3ACLT mice, the number of DRG neurons activated by knee pinch was significantly decreased in Dmp1-Rank $l^{\text {flfl }}$;Pirt-GCaMP3ACLT mice (Figure 3, F and G). The intensity of responding neurons was then analyzed. The maximum magnitude and duration of response between wild-type (WT) and conditional $\mathrm{KO}$ mice remained the same in response to mechanical force (Figure $3 \mathrm{H}$ ). Secondary allodynia assessed by von Frey test (61) showed that there was a significant decrease in the paw withdrawal thresholds (PWTs) induced by ACLT in Rank $l^{f l / f l}$ control mice by 1 week that persisted through 16 weeks (Figure 3I). Dmp1-Rank $t^{l / f l}$ mice had a significant decrease in PWT 1 week after ACLT, but PWT was soon upregulated and was similar to that of sham-surgery controls by 2 weeks (Figure 3I). Furthermore, ink blot analysis revealed a significant disparity between the percentage of right hind paw ipsilateral intensity (Figure 3, J and K) and contact area (Figure 3, J and L) of the 2 limbs at 1 month after ACLT surgery in Rank $t^{l / f l}$ controls relative to sham-surgery controls, which was not observed in Dmp1-Rank $l^{t / f l}$-ACLT mice. No significant changes were observed between $R a n k l^{f / f l}$ and Dmp1-Rank $l^{l / f l}$ mice in ipsilateral stride length or hind paw base of support (BOS) (Supplemental Figure 4, I and J). Together, these results indicate that sensory innervation induced by subchondral bone osteoclasts may mediate OA pain.

Netrin-1 secreted by osteoclasts and axonal growth. To examine the molecular mechanism by which osteoclasts regulate axonal growth, we cultured macrophages/monocytes to differentiate into osteoclasts, as evidenced by $\mathrm{TRAP}^{+}$staining and the number of nuclei (Supplemental Figure 5). The conditioned media of macrophages/monocytes and osteoclasts were collected to screen potential factors from osteoclasts that could promote axonal growth. Primary DRG neurons were collected from adult mice and cultured on the cellular side of a microfluidic culture platform, an in vitro method used widely in studies of axonal injury and regeneration by probing axons independently from cell bodies (62). The wells on the axonal side were filled with different conditioned media. Osteoclast-conditioned media induced growth of axons across the microchannels into the axonal side. However, macrophage/monocyte-conditioned media had little effect on axonal growth (Figure 4A). This finding suggests that one or more diffusible factors were secreted in the osteoclast-conditioned media and promoted axonal growth. To identify the potential secreted factor(s), we added functional blocking antibodies against Slit3, ephrinB2, Sema3A, and netrin-1 to the conditioned media. The antibody against netrin-1 inhibited the axonal growth induced by the osteoclast-conditioned media, whereas the other antibodies 
A

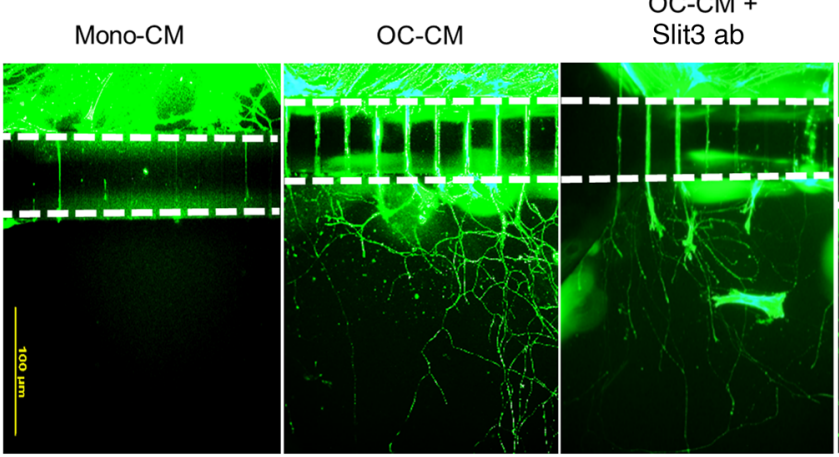

$\mathrm{OC}-\mathrm{CM}+$

$\mathrm{OC}-\mathrm{CM}+$

$\mathrm{OC}-\mathrm{CM}+$ Ephrin2B ab

Sema3A ab

Netrin-1 ab

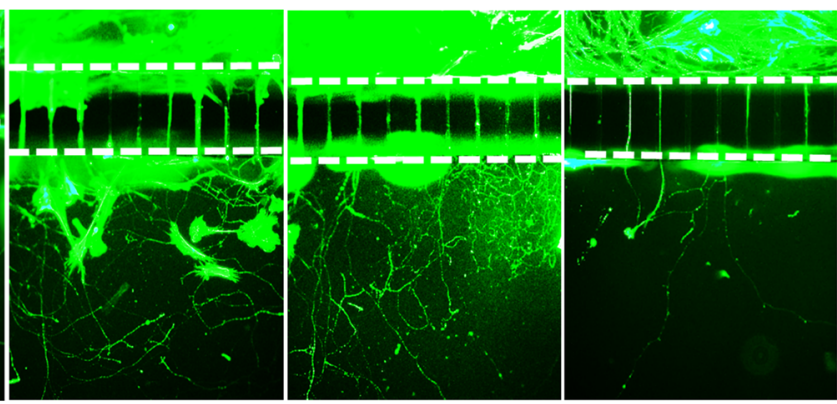

B

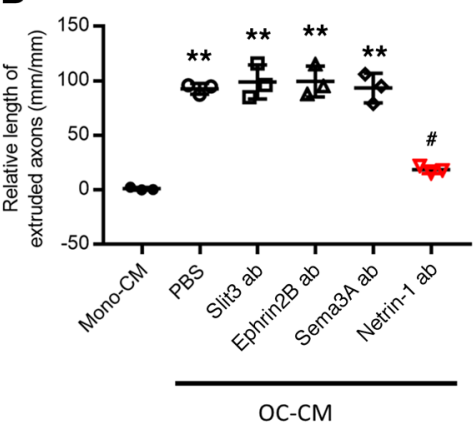

C

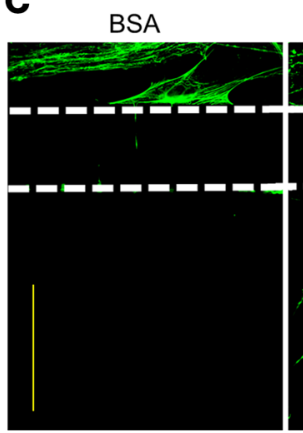

mNetrin-1

D

E

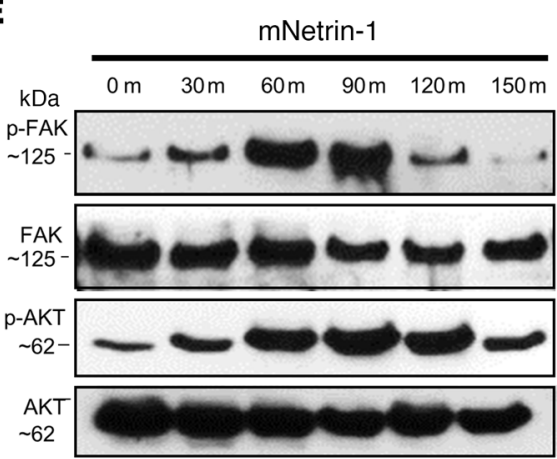

$\mathbf{F}$

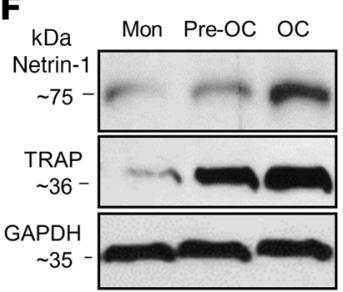

G
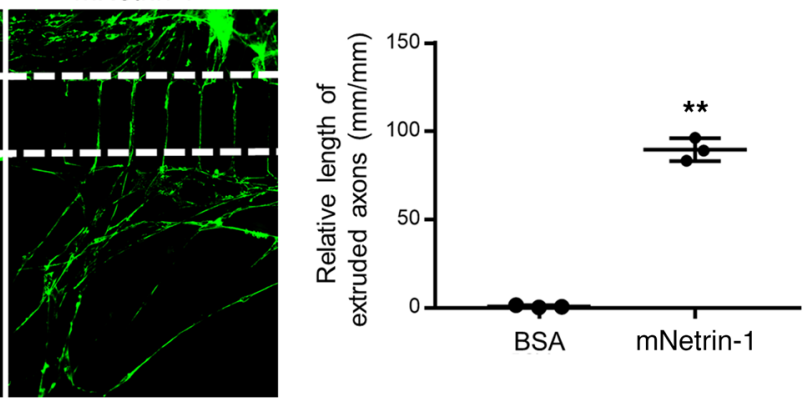

H
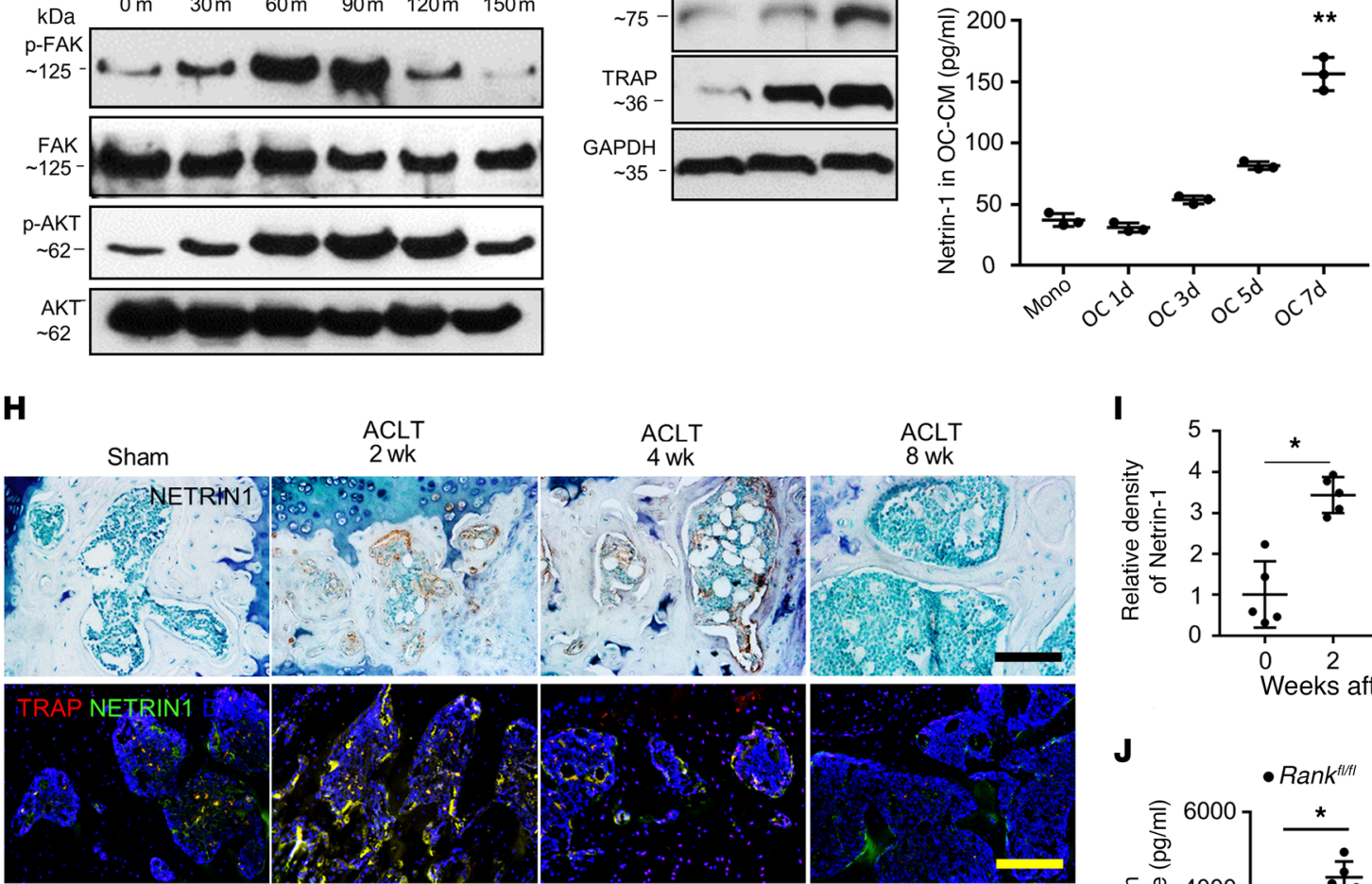

I

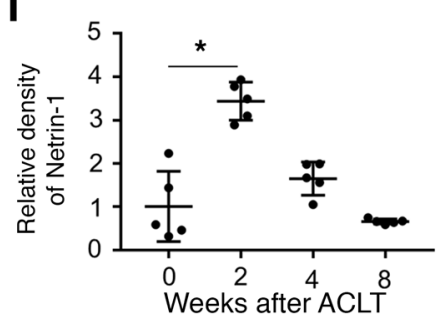

J

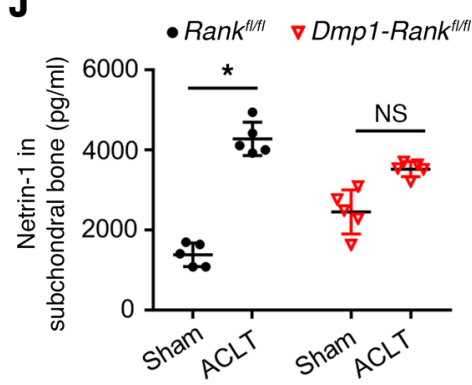


Figure 4. Netrin-1 from osteoclasts induces axonal growth. (A) Microfluidics assay of osteoclast-conditioned medium promoting DRG neuron axonal growth with treatment of functional blocking antibodies. Mono-CM, monocyte-conditioned medium; OC-CM, osteoclast-conditioned medium; ab, antibody. Scale bar: $100 \mu \mathrm{m}$. (B) Quantification of the length of axons that protruded into axonal side. ${ }^{*} P<0.01$ compared with mono-CM group; ${ }^{\#} P<0.05$ compared with OC-CM group. $n=3$ /group. (C) Microfluidics assay of recombinant mouse netrin-1 promoting DRG neuron axonal growth. Scale bar: $100 \mu \mathrm{m}$. (D) Quantification of the length of axons that protruded into axonal side. ${ }^{* *} P<0.01$ compared with BSA control group. $n=3$ /group. (E) Western blots of the phosphorylation of FAK and AKT in DRG neurons treated with netrin-1 for 0-150 minutes $(\mathrm{m})$. (F) Western blots of netrin-1 expression in monocytes, preosteoclasts, and osteoclasts. (C) ELISA analysis of netrin-1 concentration in conditioned media during osteoclast differentiation. ${ }^{* *} P<0.01$ compared with mono-CM group. (H) Immunohistochemical staining of netrin-1 and costaining of netrin-1 and TRAP in subchondral bone of WT mice at different time points after surgery. Scale bar: $100 \mu \mathrm{m}$. (I) Quantitative analysis of density of netrin-1 in subchondral bone marrow. ${ }^{*} P<0.05$ compared with the sham-operated group. (J) ELISA analysis of netrin-1 concentration in subchondral bone marrow of Rank $\left.\right|^{f / f|f|}$ and Dmp1-Rank $\left.\right|^{|f| f \mid}$ with or without ACLT surgery. ${ }^{*} P<0.05$. All data are shown as means \pm standard deviations. Statistical significance was determined by multifactorial ANOVA. NS, no significant difference.

were ineffective (Figure 4, A and B). Consistent with this finding, the addition of mouse recombinant netrin-1 peptide promoted axonal outgrowth (Figure 4, C and D).

To examine the signaling mechanisms of netrin-1-induced axon growth, we tested whether netrin-1 activates focal adhesion kinase (FAK) and PI3K/AKT pathways (63). Notably, netrin-1 induced phosphorylation of FAK and AKT at 30 minutes, peaking at 90 minutes (Figure 4E). Interestingly, netrin-1 expression was noted primarily in protein extracted from mature osteoclasts, as shown in Western blot analysis (Figure 4F) and further confirmed by enzyme-linked immunosorbent assay (ELISA) in the osteoclastconditioned media (Figure 4G). Furthermore, immunostaining demonstrated that netrin-1 colocalized with TRAP staining and was significantly higher on the bone surface 2 weeks after ACLT surgery, decreasing to baseline level at 4 and 8 weeks after ACLT surgery (Figure 4, $\mathrm{H}$ and I). We then measured the concentrations of netrin-1 in subchondral bone marrow in Dmp1-Rank $l^{t / f l}$ and Rank $l^{l / f l}$ mice. ACLT-operated Rankllf/ll control mice had increased concentrations of netrin-1 in subchondral bone marrow relative to sham-surgery Rank $l^{t / f l}$ mice (Figure $4 \mathrm{~J}$ ). The concentration of netrin-1 was higher in Dmp1-Rank $l^{l / f l}$ mice relative to Rank $l^{l / f l}$ controls but did not increase significantly after ACLT surgery relative to sham-operated controls (Figure 4J). In addition, we examined netrin-1 expression in the subchondral bone of human knee joints with $\mathrm{OA}$. There were more $\mathrm{TRAP}^{+}$osteoclasts expressing netrin-1 in osteoarthritic subchondral bone than there were in healthy controls (Figure 5, A and B, and Table 1). Taken together, these findings demonstrate that osteoclast-induced subchondral bone remodeling mediates OA pain, with a possible role for netrin-1 in promoting sensory innervation progression.

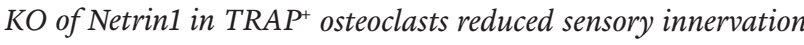
in osteoarthritic subchondral bone and OA pain. We then examined the functions of netrin-1 secreted by osteoclasts in subchondral sensory innervation in vivo. We crossbred Netrin1-floxed mice $\left(N t n^{f / f l}\right.$ mice) with Trap-Cre mice to generate Trap-Ntn $n^{f / f l}$ mice with the deletion of Netrin1 in the $\mathrm{TRAP}^{+}$cell lineage. The concentration of netrin-1 decreased significantly in the subchondral bone of Trap-Ntn fl/fl mice relative to their WT littermates with ACLT according to ELISA (Figure 6A). Additionally, in vitro Western blot assay and immunostaining showed significantly decreased netrin-1 in the subchondral bone of Trap-Ntn fl/fl mice (Supplemental Figure 6, A and B). Safranin orange and fast green staining showed similar cartilage degeneration in WT and Trap-Ntn $n^{f / f l}$ mice after ACLT (Figure 6B), as also reflected in OARSI scores (Figure 6C). The tibial subchondral bone also showed similar changes in Trap-Ntn $n^{f / f l}$ mice and WT littermates (Figure 6D and Supplemental Figure 6, C and D) after ACLT surgery. Moreover, the subchondral bone remodeling rate (as indicated by number of osterix ${ }^{+}$ osteoblast progenitors and pSMAD2 $/ 3^{+}$cells) increased similarly in $N t n^{f / f l}$ and Trap-Ntn $n^{f / f l}$ mice after ACLT (Supplemental Figure 6, G-I), suggesting that Netrin1 does not mediate OA progression. Importantly, although the number of $\mathrm{TRAP}^{+}$osteoclasts increased after ACLT in the Trap-Ntn ${ }^{f / f l}$ mice (Figure 6, E and F), the density of $\mathrm{CGRP}^{+}$sensory nerves was similar to that of sham-surgery controls (Figure 6, E and G). These findings suggest that netrin-1 secreted by osteoclasts plays an important role for sensory nerve innervation into subchondral bone.

We also measured DRG neuron activation in response to mechanical force. Trap-Ntn $n^{f / f l}$ mice were crossed with PirtGCaMP3 mice to yield Trap-Ntn ${ }^{f /-}$;Pirt-GCaMP3 mice with calcium indicator expression in DRG neurons. Because mouse Pirt (chromosome 11, NC_000077.6, 66911910.66929877) and Netrin1 (chromosome 11, NC_000077.6, 68209364.68386826, complement) are in close proximity, per the law of linkage and crossing over, no homozygous Ntn ${ }^{f / f l} ;$ Pirt-GCaMP3 mice were obtained. ELISA analysis confirmed that 1-allele deletion of Netrin1 in osteoclasts was sufficient to significantly decrease the netrin-1 concentration in osteoarthritic subchondral bone (Supplemental Figure 7C). Consistently, although some CGRP ${ }^{+}$sensory fibers could be seen in the subchondral bone of the heterozygous Netrin1-KO mice after ACLT, the density of CGRP ${ }^{+}$nerve endings (Supplemental Figure 7, A and B) and the number of activated DRG neurons (Figure 6, H and I) in Trap-Ntn ${ }^{f l-}$;Pirt-GCaMP3 mice were significantly lower than those in Ntn ${ }^{f / f l} ;$ Pirt-GCaMP3 mice after ACLT. The intensity of neuronal responses was then analyzed. The maximum magnitude and duration between $N t n^{f / f}$; Pirt-GCaMP3 and Trap-Ntn ${ }^{f l-; P i r t-G C a M P 3 ~ r e m a i n e d ~ t h e ~ s a m e ~ i n ~}$ response to mechanical force (Figure 6J).

To test whether sensory innervation in subchondral bone mediates OA pain, we measured PWTs in Trap-Ntn $n^{f / f l}$ mice. A significantly decreased PWT was sustained in $N t n^{f / f l}$ mice at 1-16 weeks after ACLT surgery (Figure 6K). However, in Trap-Ntn $n^{f / f l}$ mice, the decreased PWT did not persist, becoming upregulated after the acute phase of 1 week (Figure 6K). A similar though less effective upregulation of PWT was also seen in Netrin1 heterozygous Trap-Ntn ${ }^{f l-}$ mice (Supplemental Figure 7D). Ink blot analysis revealed a significant disparity in the percentage of right hind paw ipsilateral intensity (Figure 6, L and $\mathrm{M}$ ) and contact area (Figure 6, L and N) in WT mice after ACLT surgery that was abrogated in Trap-Ntn $n^{f l f l}$-ACLT mice. No differences in right hind paw ipsilateral stride length nor percentage of hind paw BOS were observed between $N t n^{f l / f l}$ and Trap-Ntn $n^{f / f l}$ ACTL and sham-surgery groups 
A
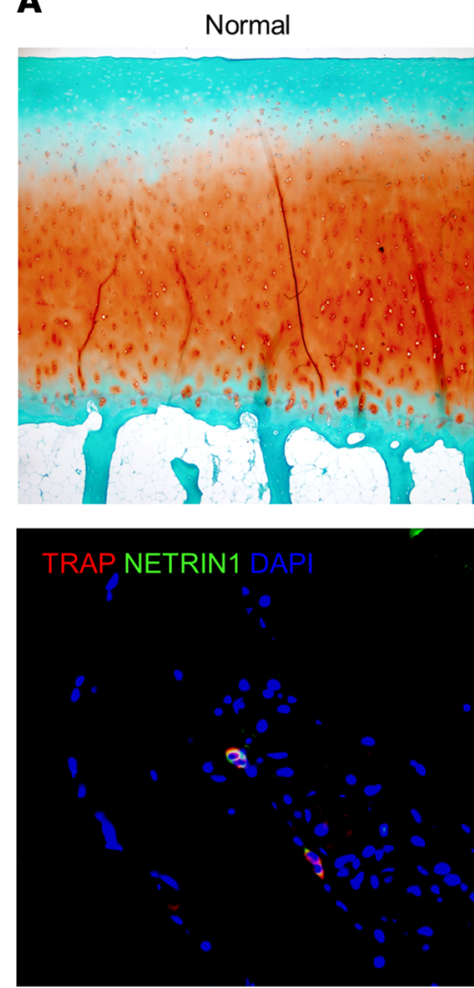

$\mathrm{OA}$
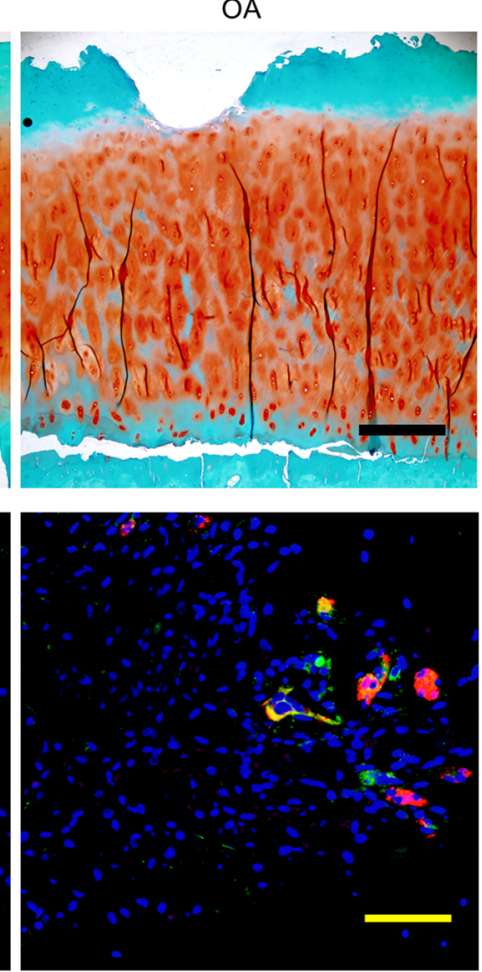

B

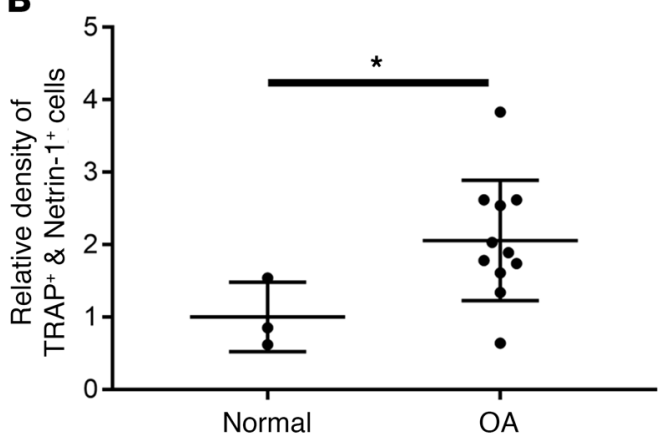

Figure 5. Osteoclast-derived netrin-1 is elevated in human OA subchondral bone. (A) Top: Safranin orange staining of human normal and OA cartilage and subchondral bone. Scale bar: $100 \mu \mathrm{m}$. Bottom: Immunofluorescence staining of TRAP and netrin-1 in human subchondral bone. Scale bar: $50 \mu \mathrm{m}$. (B) Quantitative analysis of relative intensity of TRAP and netrin-1 double-positive cells in human subchondral bone. ${ }^{*} P<0.05$, compared with healthy control by unpaired, 2-tailed Student's $t$ test.
(Supplemental Figure 6, E and F). Thus, netrin-1 secreted from osteoclast-lineage cells stimulates sensory innervation into osteoarthritic subchondral bone to mediate chronic OA pain but has no effect on OA progression.

Netrin-1 promoted sensory innervation through its receptor DCC. To identify the receptor for netrin-1 that promotes neuronal growth, we first used in vitro microfluidic assays with DRG neurons treated with scramble, anti-Dcc, or anti-Unc5 small interfering RNAs (siRNAs). Knockdown of the expression of Dcc, but not Unc5, blocked the axonal protrusion induced by osteoclastconditioned media (Figure 7A), suggesting that netrin-1 exerts its attractive functions through DCC. We next tested the requirement of DCC for sensory nerve fiber innervation into subchondral bone in vivo. We administered Ambion in vivo siRNA by tail vein injection. Knockdown of Dcc by injection of siRNA into the WT mouse tail vein did not halt the progression of OA, as indicated by similar degeneration of cartilage in the knee joint and OARSI score (Figure 7, B and C). However, the numbers of sensory fibers positive for DCC and CGRP (Figure 7, B and C) were decreased significantly in the ACLT group treated with siDcc compared with those treated with scramble siRNA. We further tested whether inhibition of sensory innervation by Ambion in vivo siDcc could ameliorate OA pain behavior. PWTs in siDcctreated ACLT mice were significantly higher than in scramble siRNA-treated ACLT mice 4 weeks after surgery and persisted through 8 weeks (Figure 7D). Gait parameters were then measured using the CatWalk gait analysis system. In scramble siRNAinoculated ACLT mice, left hind/right hind (LH/RH) paw pressure (light intensity), $\mathrm{LH} / \mathrm{RH}$ print area ratio, and swing speed were significantly decreased, which were all abrogated in siDcctreated ACLT mice (Figure 7E). Together, these findings suggest that inhibition of DCC expression in subchondral bone reduced OA pain after ACLT surgery.

Inhibition of osteoclasts by alendronate ameliorated $O A$ pain and disease progression. Bisphosphonates are an antiresorptive class of drugs that inhibit osteoclast resorptive activity and induce osteoclast apoptosis (64). The bisphosphonate alendronate (ALN) has been shown to be a potentially useful therapeutic agent for slowing the development of OA through chondroprotective effects and inhibition of subchondral bone remodeling in various surgical animal models (65-67). Compared with ACLT-induced OA, DMM-induced OA results in imbalanced joint biomechanics that lead to relatively slow disease progression and are usually considered more clinically relevant (68). Moreover, it has been demonstrated that standard analgesics can reverse pain in DMM mice, making this model ideal to test the effect of analgesics on OA pain development (10). Thus, for our intervention studies, the DMM model was used to test the effect of ALN on OA pain relief. Time-course analysis of netrin-1 levels in subchondral bone revealed that the density of netrin-1 staining was significantly higher 2 weeks after DMM compared with the sham group and peaked at 4 weeks (Supplemental Figure 8, A and B). This seems to be a delayed response compared with that in subchondral bone of ACLT mice, which is consistent with slower disease progression in the DMM model. Similar to the results in ACLT mice, immunostaining of CGRP in DMM mice showed an increasing distribution of peptidergic nociceptive nerve fibers adjacent to the trabecular bone surface beginning 1 week after surgery (Supplemental Figure $8, \mathrm{~A}$ and $\mathrm{C})$. The numbers and density of nerve endings remained increased at 8 weeks after DMM surgery. Eight weeks after DMM surgery, vehicle-treated mice had loss of safranin orange staining, fibrous/defective surface cartilage, and significantly elevated 
Table 1. Information for the human samples

$\begin{array}{lll} & \text { Normal } & \text { OA } \\ \text { Sample Size } & 3 & 11 \\ \text { Sex } & 2 \mathrm{M} / 1 \mathrm{~F} & 6 \mathrm{M} / 5 \mathrm{~F} \\ \text { Height }(\mathrm{cm}) & 172 \pm 3.4 & 169 \pm 2.9 \\ \text { Body weight }(\mathrm{kg}) & 69 \pm 5.8 & 71 \pm 3.7 \\ \text { Age } & 33.2 \pm 4.5 & 56.8 \pm 2.7 \\ \text { BMI } & 22.4 \pm 2.7 & 23.8 \pm 1.6 \\ \text { Various deformity (degree) } & \mathrm{N} / \mathrm{A} & 10.5 \pm 2.7 \\ \text { KL stage } & 0 & 2.9 \pm 0.7 \\ \text { KSS score } & \mathrm{N} / \mathrm{A} & 53.1 \pm 7.9\end{array}$

KL, Kellgren and Lawrence classification; KSS, Knee Society score; N/A, not applicable.

OARSI scores (Figure 8, A and B). Similar to the ACLT OA model, DMM mice also had increased $\mathrm{TRAP}^{+}$osteoclasts and $\mathrm{CGRP}^{+}$ sensory nerves (Figure 8, A and B) in the subchondral bone. ALN treatment attenuated $\mathrm{OA}$ progression, the number of $\mathrm{TRAP}^{+}$ osteoclasts, and the number of $\mathrm{CGRP}^{+}$sensory nerves (Figure 8, $A$ and B). ALN treatment also reduced the netrin-1 staining in subchondral bone (Figure 8C). Moreover, the decreased PWT after DMM was attenuated in the ALN-treated DMM mice at 4 weeks, which persisted at 8 weeks relative to vehicle-treated mice (Figure 8D). Using CatWalk gait analysis, we found that DMM surgery resulted in similar decreases of $\mathrm{LH} / \mathrm{RH}$ print area, duty cycle, and swing speed and increased swing phase. These were prevented by ALN treatment (Figure 8E), suggesting that inhibition of osteoclast activity by ALN ameliorates OA pain.

\section{Discussion}

Current OA pain management strategies have limited therapeutic effects, and progressive pathological joint changes are observed frequently with these treatments. The 2012 American College of Rheumatology guidelines recommend analgesics and nonsteroidal antiinflammatory drugs as the first-line pharmacologic therapies for OA of the hand, hip, and knee (69). However, these drugs provide insufficient and unsustained pain relief with considerable adverse effects. Our previous study revealed that excessive activation of TGF- $\beta 1$ during subchondral bone remodeling recruits mesenchymal stem cells for aberrant bone formation and angiogenesis, which is a key step in the pathogenesis of OA $(39,70)$. In the current study, we report that osteoclastinitiated subchondral bone remodeling mediates OA pain, with a possible role for osteoclast-secreted netrin-1. For the first time to our knowledge, we revealed that nociceptors are generated during aberrant subchondral bone remodeling in the early phase of OA. Our findings suggest that inhibition of aberrant subchondral bone formation can reduce sensory innervation and attenuate articular cartilage degeneration.

Articular cartilage and subchondral bone are not only a mechanical unit but also a biological functional unit (71). The structural alterations of subchondral bone in OA are believed to enhance its capacity for crosstalk with articular cartilage. In this study, we observed significant protection of articular cartilage from degeneration in Dmp1-Rank $k^{l / f l}$ mice, suggesting that active subchondral bone remodeling in response to abnormal mechanical loading is critical for OA progression. Specifically, the decreased number of osteoclasts in Dmp1-Rank $l^{f / f l}$ mice led to significantly reduced hyperexcitability of DRG neurons in response to mechanical stimulation applied to osteoarthritic joints. Consistent with previous reports $(26,28)$, an increased number of $\mathrm{CGRP}^{+}$sensory nerves was seen in the synovium of both Dmp1-Rankll/fl (Supplemental Figure 9, A and B) and Trap-Ntn ${ }^{f / f l}$ (Supplemental Figure 9, $\mathrm{C}$ and D) mice after OA surgery in our study. However, the density of $\mathrm{CGRP}^{+}$neurofilaments in subchondral bone was decreased in Dmp1-Rank $l^{l / f l}$ and Trap-Ntn ${ }^{f / f l}$ mice. These results further indicate that osteoclasts in aberrant subchondral bone remodeling could be an important origin of OA pain. The various methods used to measure pain in this study are complementary. We examined mechanical hypersensitivity to von Frey filament stimulation applied to hind paws in OA animals. Mechanical hypersensitivity of hind paws may represent the secondary hyperalgesia developed after OA. Activation and sensitization of nociceptive neurons (peripheral sensitization) may contribute to the hyperalgesia at the knee joint (e.g., pinch-evoked pain hypersensitivity at the joint and movement-induced behavioral changes). Indeed, our in vivo GCaMP3 imaging experiments also indicated a hypersensitivity of DRG neurons to stimulation at the knee. It is important to note that continued nociceptive input may also induce central sensitization (e.g., increase of spinal dorsal horn neuron excitability) that would amplify the afferent input from the diseased joint and also lead to secondary hyperalgesia in other somatic body regions (e.g., hind paws). Central sensitization has been suggested by many clinical and animal studies to contribute to the lack of direct correlation between nociceptor activation and the pain. Primary hyperalgesia in osteoarthritic knees has recently been investigated by measuring withdraw threshold in response to direct knee pinching or press using a pressure application measurement (PAM) device in a mouse DMM model (72). Future studies integrating this method of pain measurement would further complement our current study.

The increased remodeling rate in subchondral bone is initiated by osteoclasts and is a known pathological feature of $\mathrm{OA}$, particularly during the early stage of disease (38). Osteoclastlineage cells are the principal, if not exclusive, bone-resorbing cells essential for bone remodeling and skeletal development. Osteochondral junctions have long been implicated as early sites of new blood vessel growth, which is accompanied by extensions of sympathetic and sensory nerves in $\mathrm{OA}(28,29)$. Blood vessel and nerve growth are linked by common pathways activated during the release of proangiogenic factors (73). Our study has identified a possible role for netrin- 1 secreted by osteoclasts during aberrant subchondral bone remodeling in inducing sensory innervation and OA pain. In addition to its role in axon guidance, netrin-1 has been suggested to be a potent vascular mitogen (74-76). Netrin-1 was found to promote angiogenesis by controlling endothelial cell migration (75), tubal formation (77), and apoptosis blockade (78). Our previous study revealed that preosteoclasts secrete plateletderived growth factor-BB during bone remodeling to induce angiogenesis coupled with osteogenesis during bone formation (52). Thus, osteoclast-lineage cells may promote both nerve and vessel growth in osteoarthritic subchondral bone, leading to disease progression and pain. 

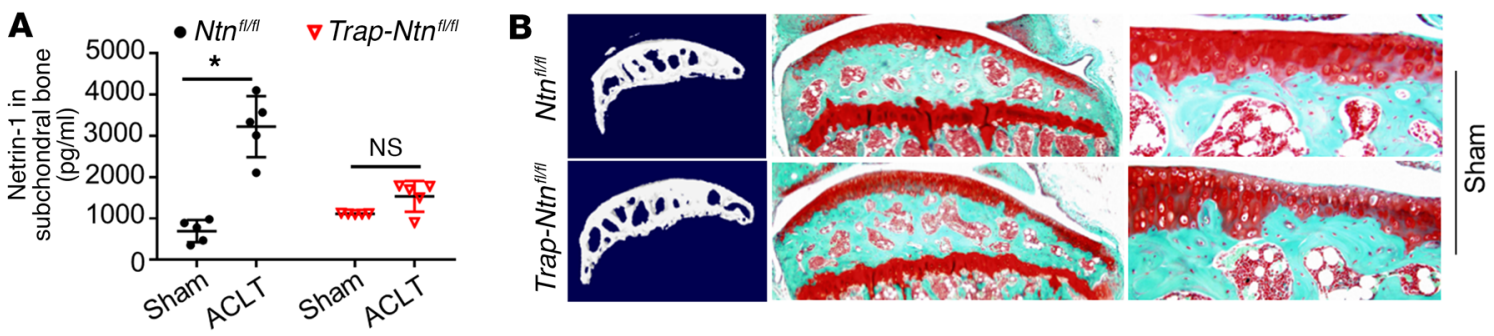

C
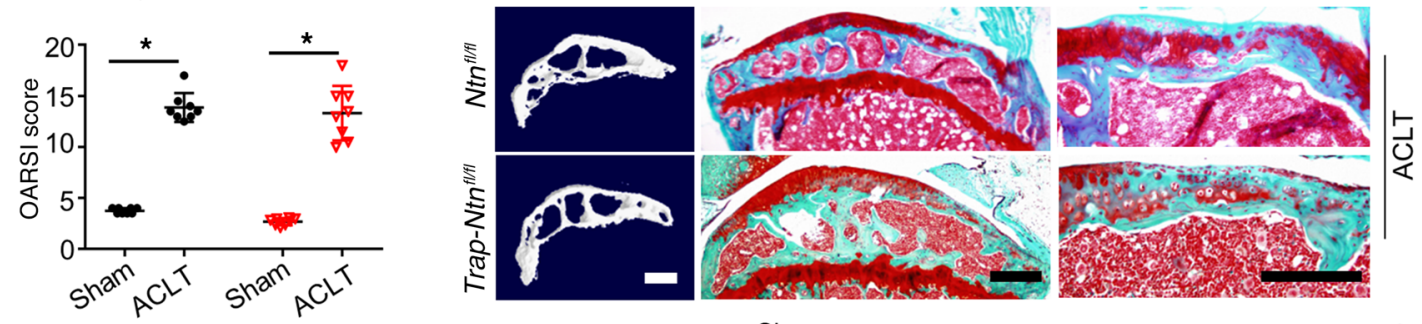

D

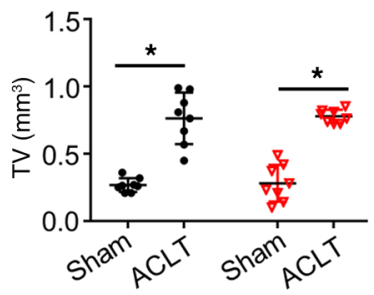

E

Sham ACLT
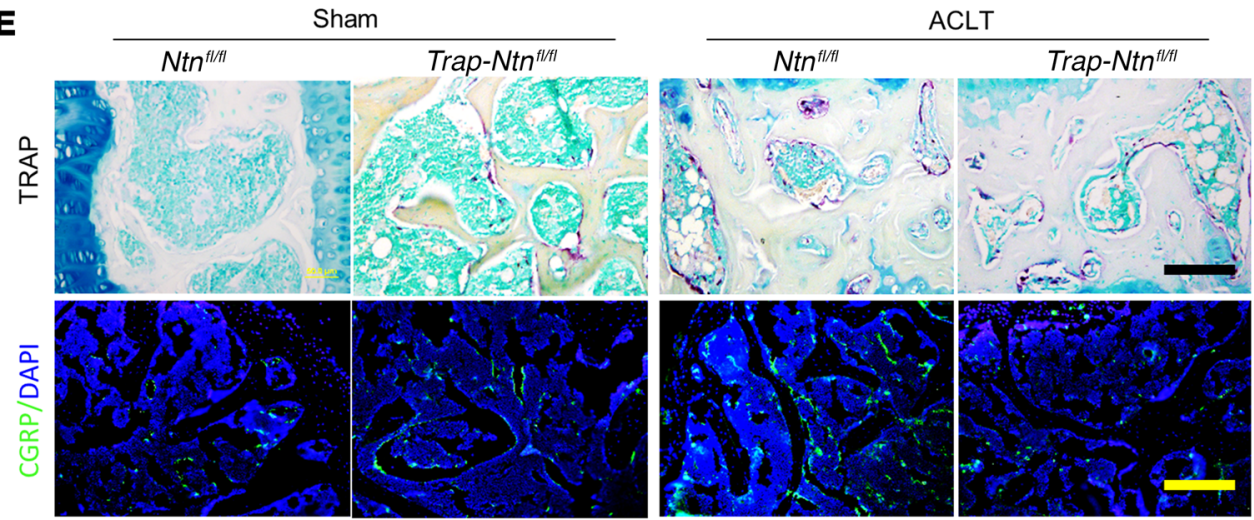

$\mathbf{F}$

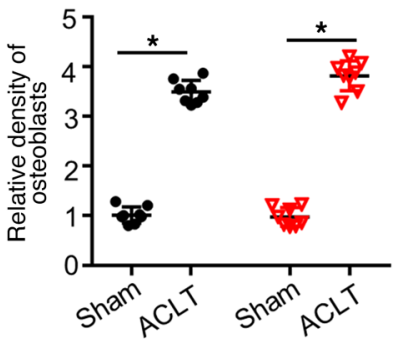

H

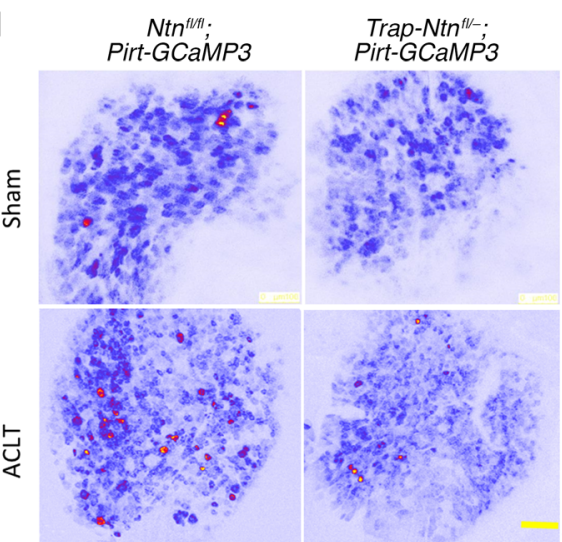

$\mathbf{L}$

Trap-Ntn ${ }^{t / f i}$

G

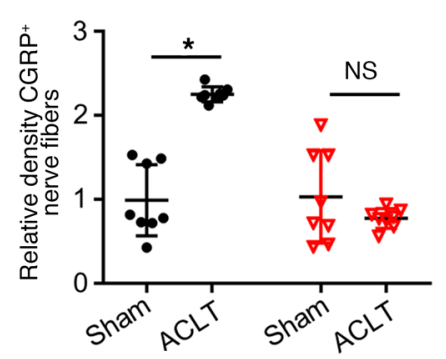

$\frac{\varepsilon}{\frac{\varepsilon}{5}}$
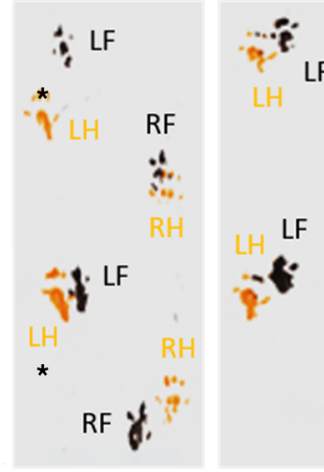

LF RF

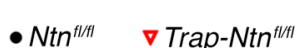

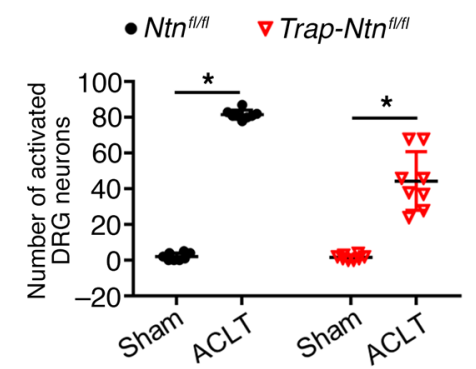

J

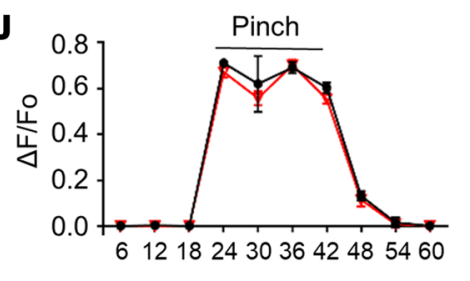

$\mathbf{K}^{\bar{\pi}}$
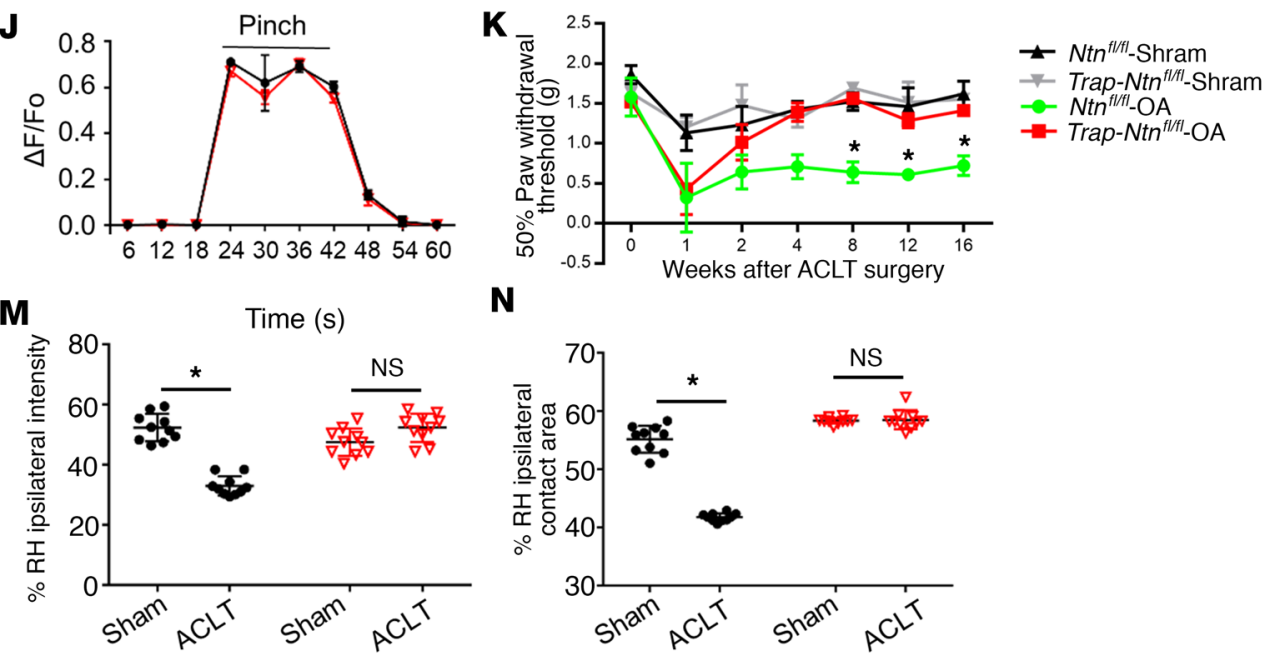
Figure 6. Knockout of Netrin1 in osteoclast-lineage cells reduces sprouting of CGRP' sensory nerves in subchondral bone and ameliorates OA pain. (A) ELISA analysis of netrin-1 concentration in subchondral bone marrow of $N t n^{f / f l}$ and Trap-Ntn $n^{f l / f l}$ mice with or without ACLT surgery. $n=5$ /group. (B) Left: 3-Dimensional $\mu \mathrm{CT}$ image of the tibial subchondral bone medial compartment (sagittal view) of $\mathrm{Ntn}^{\text {fl/fl}}$ and $\mathrm{Trap}^{\mathrm{N}} \mathrm{Nn^{f/fl }}$ with or without ACLT surgery. Middle and right: Safranin orange and fast green staining of articular cartilage in sagittal sections of tibial medial compartment of mice. Scale bars: $1 \mathrm{~mm}$ (left), $500 \mu \mathrm{m}$ (middle), and $100 \mu \mathrm{m}$ (right). (C) OARSI scores 8 weeks after surgery. $n=8$ /group. (D) Quantitative analysis of total tissue volume (TV) in subchondral bone determined by $\mu \mathrm{CT}$. $n=8$ /group. (E) TRAP staining (top, magenta) and immunofluorescence analysis of CGRP ${ }^{+}$ sensory nerve fibers (bottom, green) in mouse tibial subchondral bone after ACLT surgery. Scale bars: $50 \mu \mathrm{m}$. (F and $\mathbf{G}$ ) Quantitative analysis of relative density of $\mathrm{TRAP}^{+}$osteoclasts and $\mathrm{CGRP}^{+}$nerve fibers in subchondral bone marrow. (H) In vivo calcium imaging in whole L4 DRG primary sensory neurons after mechanical press to knees of $\mathrm{Ntn}^{f / / f l}$;Pirt-GCaMP3 and Trap$N t n^{f l /} ;$; Pirt-GCaMP3 ACLT mice. Scale bars: $50 \mu \mathrm{m}$. (I) Number of neurons activated by mechanical press. (J) $\Delta F / F o$ for neurons in a representative DRG responding to approximately 20 -g knee pinch in $\mathrm{Ntn}^{f / f f l}$ (black) and Trap-Ntn ${ }^{f /-}$ (red) mice after ACLT. (K) Paw withdrawal threshold (PWT) was tested at the right hind paw of $N t^{f l / f l}$ and $T r a p-N t n^{f l / f l}$ mice with

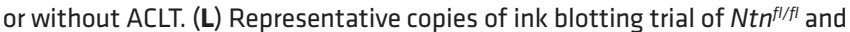
Trap-Ntn ${ }^{f l / f l}$ mice after ACLT surgery on right knees. RH, right hind (orange); $\mathrm{LH}$, left hind (orange); RF, right front (black); LF, left front (black). (M and $\mathbf{N})$ Percentage RH ipsilateral intensity $(\mathbf{M})$ and percentage $\mathrm{RH}$ ipsilateral contact area (N) determined by Imagej software. $n=10 /$ group. All data are shown as means \pm standard deviations. ${ }^{*} P<0.05$ by multifactorial ANOVA. NS, no significant difference.

We acknowledge that other mechanisms may exist for osteoclast activation-induced OA pain. For example, osteoclasts are believed to play multiple roles in cancer-associated bone pain (CABP) (79). Specifically, osteoclasts secrete protons from bone resorption sites via $\alpha 3$ vacuolar proton-ATPase acidifying the extracellular bone microenvironment. Acidosis is algogenic for nociceptive sensory neurons that innervate into bone. Acidic environments upregulate and activate $\mathrm{pH}$-sensitive acid-sensing nociceptors, the transient receptor potential channel-vanilloid subfamily member 1 (TRPV1), and acid-sensing ion channels (ASIC3) to evoke CABP. In our study, we also observed activation of osteoclasts in the subchondral bone during early stages of OA. We think the activation of osteoclasts is bifunctional. On one hand, osteoclast-secreted netrin-1 facilitates peptidergic neurite growth. On the other hand, the osteoclastic resorption may also create an acidic environment in subchondral bone that peripherally sensitizes the nociceptive neurons. Moreover, netrin-1 was also found to activate TRPV1 in dorsal horn neurons (80). Thus, it is possible that netrin-1 itself could also peripherally sensitize nociceptive neurons.

Neuroanatomical and molecular characterization of nociceptors demonstrates the heterogeneity of C-fibers (81). The peptidergic subpopulation of nociceptors releases neuropeptides, such as substance P and CGRP, and expresses tropomyosin receptor kinase A. The nonpeptidergic subpopulation of nociceptors expresses the c-Ret receptor. A large percentage of the c-Ret-positive population also binds the isolectin IB4 and expresses $\mathrm{G}$ protein-coupled receptors of the Mrg family (82). Our retrograde-labeling data showed a significant increase in the number of $\mathrm{CGRP}^{+}$nociceptors that newly innervat- ed into osteoarthritic subchondral bone marrow. Immunofluorescence studies showed that the density of NF200, PGP9.5, and $\beta$ tubulin in subchondral bone marrow was not altered by ACLT. We observed that the density of $\mathrm{CGRP}^{+}$and other nociceptive nerve endings (e.g., P2X3 and PIEZO2) increased, while $\mathrm{PGP} 5^{+}$nerve density remained unchanged in osteoarthritic subchondral bone. Protein gene product (PGP), also known as ubiquitin carboxy-terminal hydrolase 1 (UCHL1), is a pan neuronal marker that labels most peptidergic and nonpeptidergic, nociceptive, and non-nociceptive neurons. Our observation suggests that innervation of other non-CGRP-expressing neuronal populations might decrease, leaving the total density of PGP9. $5^{+}$nerves unchanged. Indeed, a recent study (83) using a UCHL1-eGFP reporter line found that approximately $64 \%$ of bright $\mathrm{DRG} \mathrm{eGFP}^{+}$neurons expressed CGRP, while the remaining approximately $36 \%$ of neurons did not, indicating that $\mathrm{CGRP}^{+}$subpopulations exist in PGP9.5expressing neurons. We acknowledge that determining the dynamic changes in subpopulations of neurons that innervate into subchondral bone in response to ACLT will require different genetic methods in our future work. Our findings agree with the observation in recent studies that the percentage of CGRP ${ }^{+}$ neurons innervating subchondral bone was significantly augmented after OA induction (84). Yet, the way in which these nociceptive neurons innervate subchondral bone marrow during OA progression remains to be investigated.

Mice deficient in Netrin1 exhibited less OA pain and minimal alterations in gait, even though they developed rapid proteoglycan loss in articular cartilage, as did WT mice. Netrin1 conditional $\mathrm{KO}$ mice showed increases in subchondral bone volume and trabecular pattern factor after OA similar to those seen in WT mice. Accordingly, inhibition of innervation of $\mathrm{CGRP}^{+}$sensory fibers in the subchondral bone reduced OA pain but did not affect subchondral bone remodeling or articular cartilage degeneration, suggesting a dissociation between pain perception and joint destruction. An extreme example of this dissociation is Charcot's joint, a process marked by bony destruction, bone resorption, and eventual deformity caused by loss of sensation (85). One well-accepted explanation of this pathogenesis is neurotrauma. Loss of peripheral sensation and proprioception leads to repetitive microtrauma to the joint (86). Mediero and colleagues reported a similar increase of netrin-1 expression during osteoclast differentiation, with accompanying autocrine and paracrine stimulation of UNC5B to promote osteoclast differentiation (48). Interestingly, our Netrin1 conditional $\mathrm{KO}$ mice have a similar subchondral bone remodeling rate as that of WT mice. This could be attributable to different microenvironments in subchondral bone versus diaphyseal bone, especially in pathological conditions such as OA, in which a combination of cytokines, chemokines, and inflammatory factors affect osteoclast/osteoblast differentiation in joints locally. Netrin-1 has been shown to bind/interact with various receptors, including DCC, UNC5 homologs, and the adenosine A2B receptor. In agreement with other studies, our results showed that netrin-1 promoted DRG neuron axonal outgrowth and subchondral bone sensory innervation through the receptor DCC. The adenosine A2B receptor has been shown to bind directly to 
$\mathbf{A}$
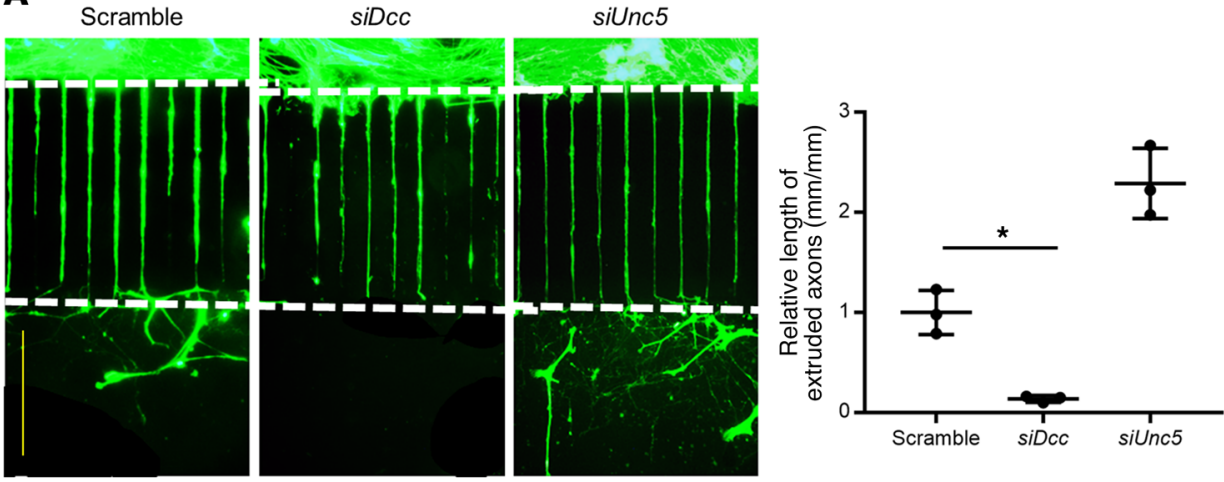

B
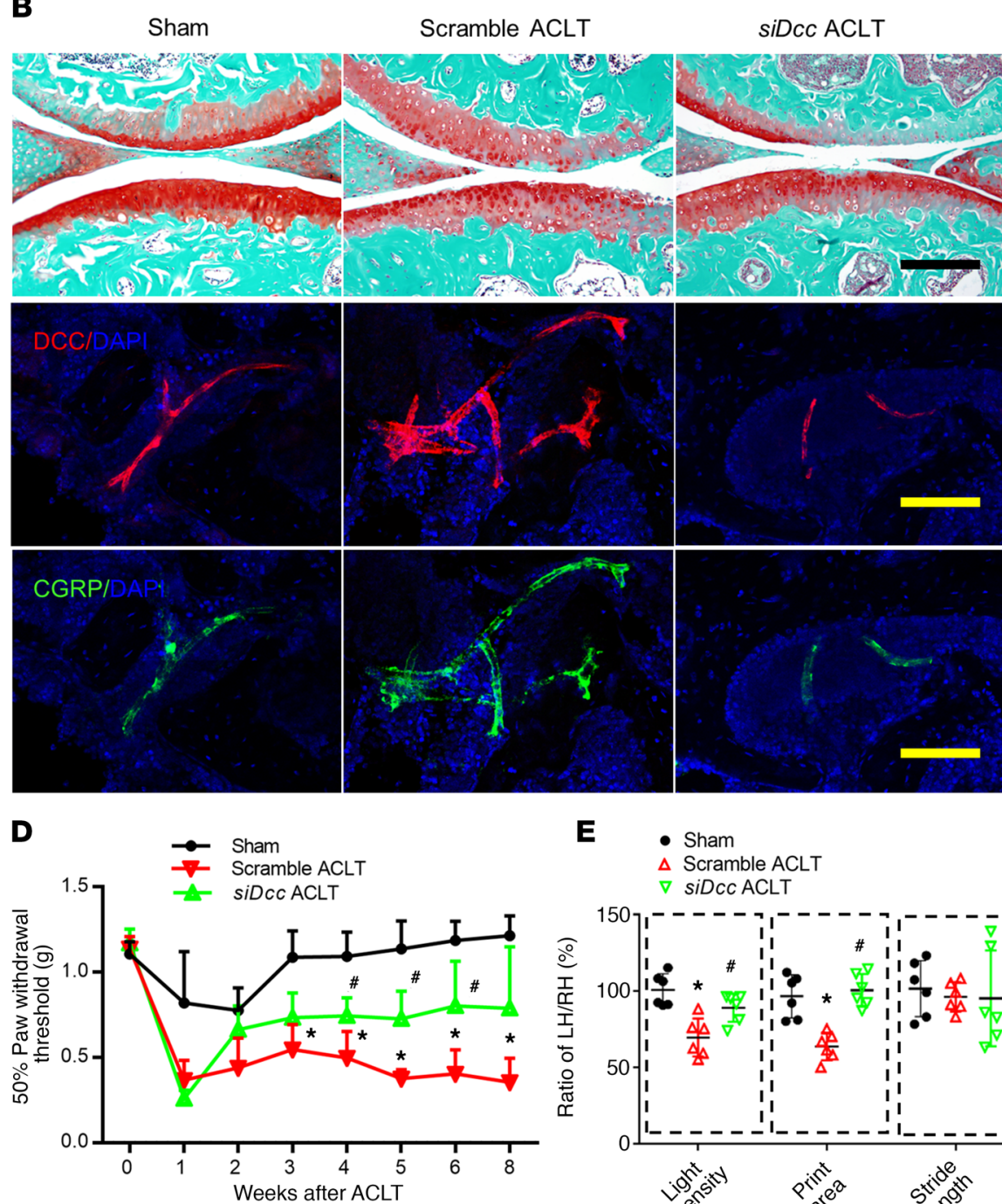

E

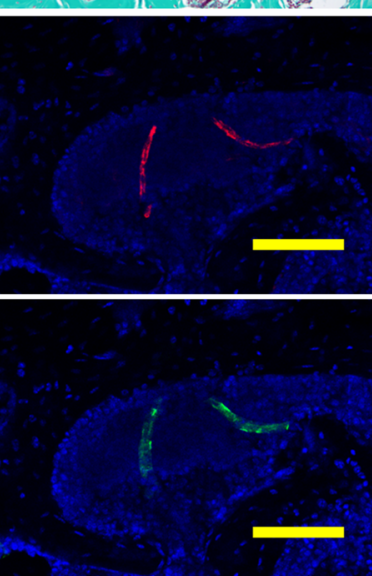

- Sham

$\triangle$ Scramble ACLT

$\nabla$ siDcc ACLT
C
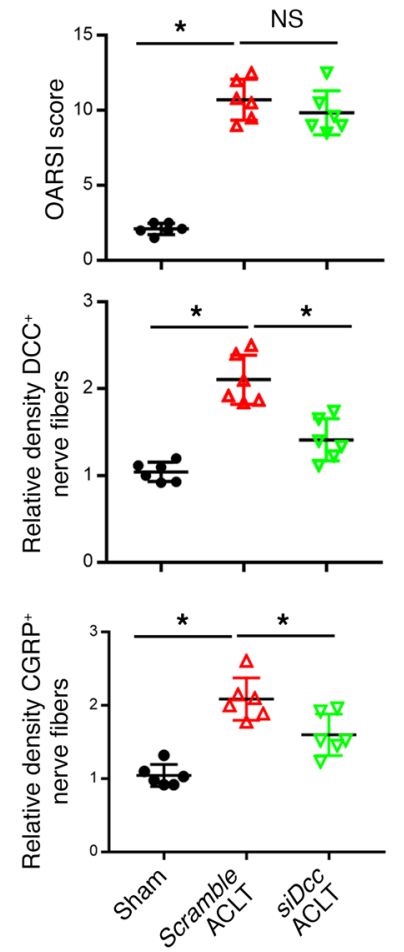

Figure 7. In vivo silencing of murine Dcc mRNA by siRNA systemic administration reduced CGRP+ sensory nerve subchondral bone innervation and OA pain. (A) Microfluidics assay of osteoclast-conditioned medium promoting DRG neuron axonal growth with treatment of siDcc and siUnc5. Scale bar: $100 \mu \mathrm{m}$. ${ }^{*} P<0.05$. (B) Top: Safranin orange and fast green staining of articular cartilage in sagittal sections of the tibial medial compartment of mice. Scale bar: $100 \mu \mathrm{m}$. Immunofluorescence analysis of $\mathrm{DCC}^{+}$(middle, red) and CGRP ${ }^{+}$(bottom, green) sensory nerve fibers in mouse tibial subchondral bone 4 weeks after surgery. Scale bars: $50 \mu \mathrm{m}$. (C) Quantitative analysis of OARSI score (top), relative density of DCC ${ }^{+}$(middle), and CGRP+ (bottom) nerve fibers in subchondral bone marrow. ${ }^{*} P<0.05$. NS, no significant difference. (D) Paw withdrawal threshold (PWT) was tested at the left hind paw of sham, scramble ACLT, and siDcc ACLT mice at different time points after surgery. ${ }^{*} P<0.05$, compared with sham mice; ${ }^{\#}<0.05$, compared with ACLT-operated and scramble siRNA-administered mice. (E) Variations in the ipsilateral and contralateral hind limbs of gait parameters obtained from CatWalk analysis. ${ }^{*} P<0.05$, compared with sham mice; ${ }^{\#} P<0.05$, compared with ACLT-operated and scramble siRNA-administered mice. Statistical significance was determined by multifactorial ANOVA, and all data are shown as means \pm standard deviations. 
A
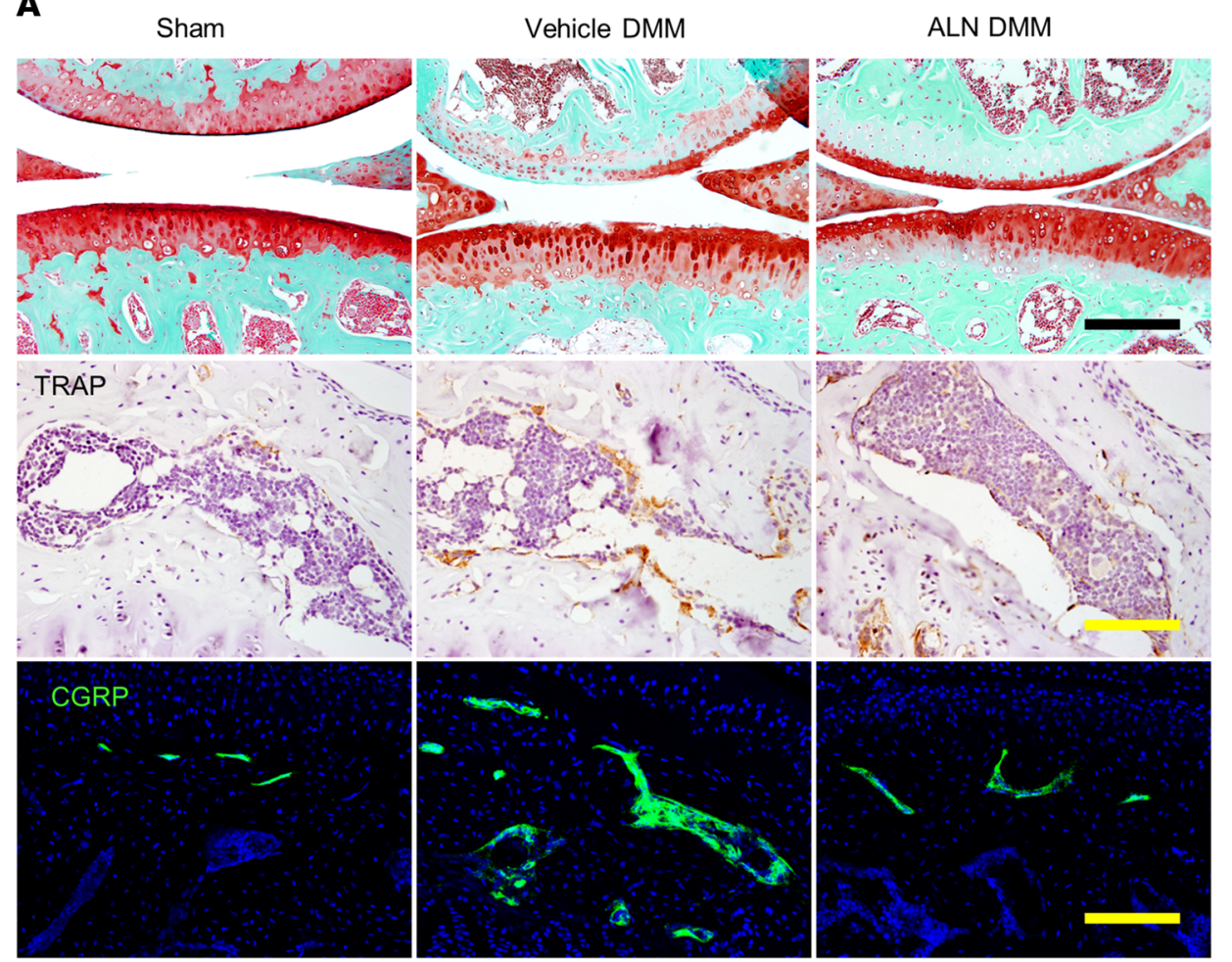

C
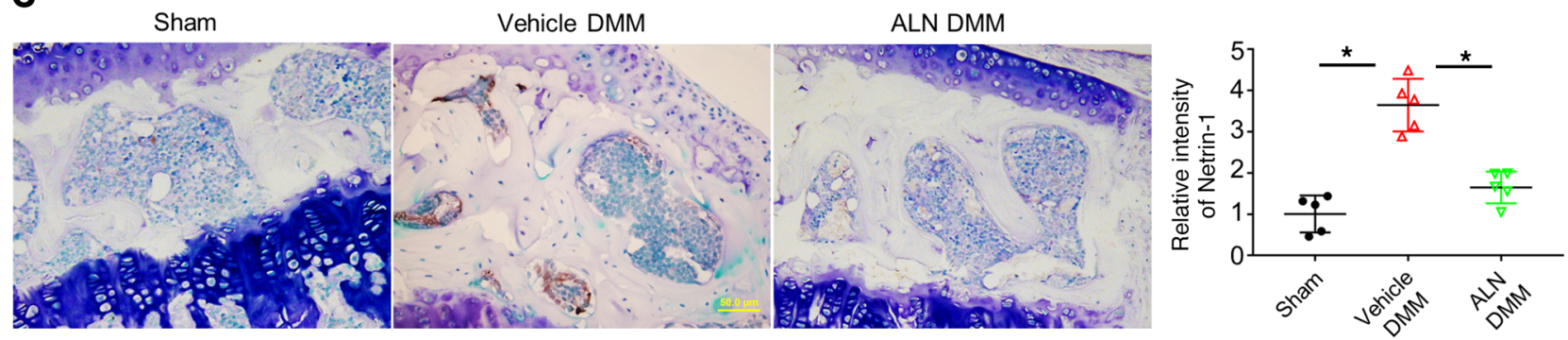
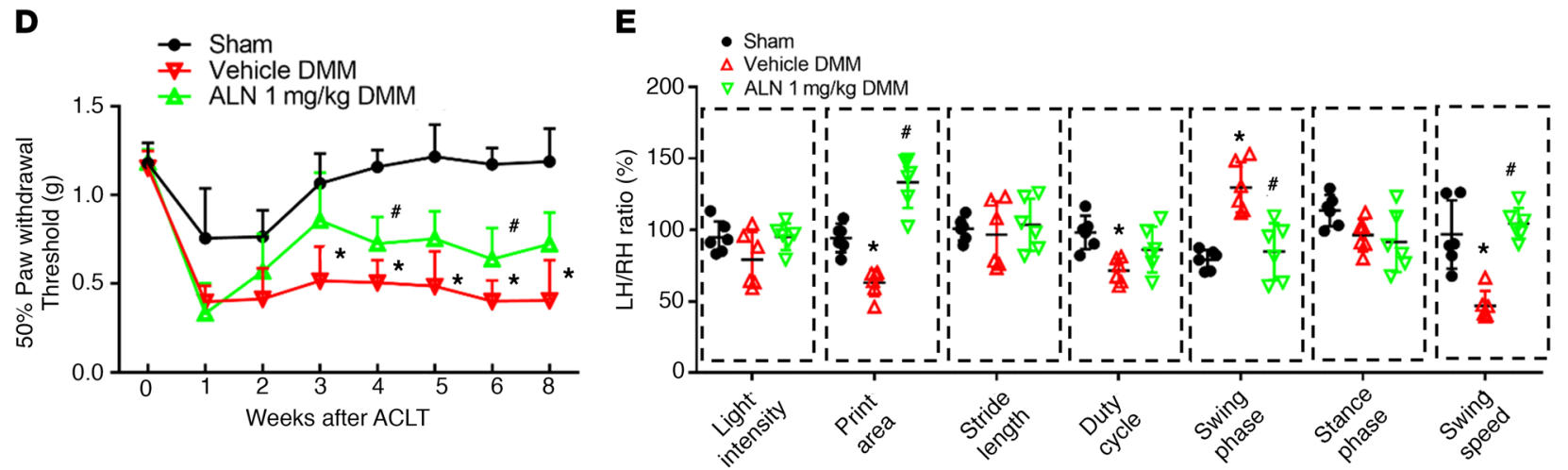

Figure 8. Effect of alendronate on DMM-induced OA pain. (A) Top: Safranin orange and fast green staining of articular cartilage in sagittal sections of the tibial medial compartment of mice. Scale bar: $100 \mu \mathrm{m}$. Middle and bottom: Immunohistochemical analysis of TRAP (middle) and immunofluorescence analysis of CGRP+ (bottom, green) sensory nerve fibers in mouse tibial subchondral bone after DMM surgery. Scale bars: $50 \mu \mathrm{m}$. (B) Quantitative analysis of OARSI score (top), relative density of TRAP ${ }^{+}$osteoclasts (middle), and CGRP+ (bottom) nerve fibers in subchondral bone marrow. ${ }^{*} P<0.05$. (C) Immunohistochemical staining and quantification of netrin-1 in subchondral bone of sham-operated mice and DMM-operated mice treated with either vehicle or ALN. Scale bar: $50 \mu \mathrm{m}$. ${ }^{*} P<0.05$. (D) Paw withdrawal threshold (PWT) was tested at the left hind paw of sham, vehicle DMM, and ALN ACLT mice at different time points after surgery. ${ }^{*} P<0.05$, compared with sham mice; ${ }^{\#} P<0.05$, compared with $D M M$-operated and vehicle-administered mice. (E) Variations in the ipsilateral and contralateral hind limbs of gait parameters obtained from CatWalk analysis. ${ }^{*} P<0.05$, compared with sham mice; ${ }^{\#} P<0.05$, compared with DMM-operated and vehicle-administered mice. Statistical significance was determined by multifactorial ANOVA, and all data are shown as means \pm standard deviations. 
DCC and function as a netrin-1 co-receptor (87). In addition, recent studies have reported that netrin-1 signaling through the adenosine A2B receptor inhibits diabetic nephropathy (88) and hypoxia-induced inflammatory cell infiltration into mucosal organs (89). Inhibition of adenosine receptors by caffeine has been used frequently as an adjuvant analgesic in combination with nonsteroidal antiinflammatory drugs or opioids $(90,91)$. Thus, it would be of interest in future studies to further examine the expression of the adenosine $\mathrm{A} 2 \mathrm{~B}$ receptor in the peripheral nervous system and its involvement in netrin-1-induced axonal outgrowth in osteoarthritic subchondral bone.

The inhibition of excessive TGF- $\beta 1$ activity or osteoclast bone resorption, such as by using bisphosphonate, may interrupt aberrant subchondral bone remodeling and reduce innervation of $\mathrm{CGRP}^{+}$sensory fibers in the subchondral bone to attenuate OA pain. Indeed, bisphosphonates have been tested in OA clinical trials (92-95) and achieved some beneficial effects on articular cartilage and marrow lesions, improved Western Ontario and McMaster Universities Osteoarthritis Index pain scores, and decreased prevalence of subchondral bone marrow lesions. However, the reported effects of bisphosphonates have been mixed (96). The lack of efficacy in some studies may be explained by the heterogeneity of pathogenesis. Our previous study showed that uncoupled aberrant subchondral bone formation led to articular cartilage degeneration. Active subchondral bone resorption releases excessive active TGF- $\beta 1$, which recruits mesenchymal stem cells to the subchondral bone marrow for aberrant bone formation along with type $\mathrm{H}$ vessel formation during the early stage of OA $(39,70)$. During the middle and late stages of OA, uncoupled abnormal bone formation has largely finished, with limited osteoclast activity (39). Accordingly, the optimal time for bisphosphonate treatment would be during the early stage of OA because this is the period when sensory innervation is induced by osteoclasts.

Given the increasing incidence of $\mathrm{OA}$ and the insufficient control of $\mathrm{OA}$ pain by current available medication, better understanding of the mechanisms of OA pain would potentially help develop more effective analgesics. Our study has determined that aberrant subchondral bone remodeling initiated by osteoclasts induces sensory innervation, with a possible role of netrin-1. Inhibition of osteoclast activity by ALN modifies aberrant subchondral bone remodeling and reduces innervation and pain behavior at the onset of OA. Our study suggests that intervention of the axonal guidance molecules (e.g., netrin-1) derived from aberrant subchondral bone remodeling may have therapeutic potential for OA pain.

\section{Methods}

Mice. We purchased C57BL/6J (WT) male mice from Charles River Laboratories. We anesthetized the mice at 2 months of age with ketamine and xylazine and then transected the ACL surgically to induce mechanical instability-associated OA of the right knee. Sham operations were performed on other groups of mice. In the sham groups, the knee capsule and infrapatellar fat pad were incised but no ACLT was performed. For the time-course experiments, mice were euthanized at $0,1,2,4$, or 8 weeks after surgery ( $n=8$ per group). DMM surgery was performed in the left knees of mice. Briefly, the surgery started with a 3-mm longitudinal incision over the distal patella to the proximal tibial plateau. The anterior medial meniscotibial ligament was identified and resected with the blade directed proximolaterally to destabilize the medial meniscus. Sham surgery followed the same procedure to expose the anterior medial meniscotibial ligament, but the ligament was left intact. Mice were not administered analgesia after surgery. ALN was injected intraperitoneally 3 times per week at a dose of $1 \mathrm{mg}$ / $\mathrm{kg}$ for 8 weeks after DMM surgery.

CatWalk analysis. Gait parameters of freely moving mice were measured using the CatWalk gait analysis system (Noldus Information Technology) as described previously (97). Briefly, the CatWalk instrument consists of an enclosed walkway with a glass plate floor, a fluorescent lamp that emits light inside the glass plate, a highspeed color video camera, and recording and analysis software to assess the gait of rodents. Each mouse was placed individually in the CatWalk walkway and allowed to walk freely and traverse from one side to the other of the walkway. Mice were trained as described previously (98). The recordings were made when the room was completely dark, except for the light from the computer screen. Where the mouse paws made contact with the glass plate, light was reflected down and the illuminated contact areas recorded with a high-speed color video camera that was positioned under the glass plate and connected to a computer running the CatWalk software, v7.1. The software automatically labeled all areas containing pixels above the set threshold (7 pixels). These areas were identified and assigned to the respective paws. The recording generated a wide range of parameters, the following 7 of which were analyzed: paw pressure, paw print area, stance phase, swing phase, duty cycle, stride length, and swing speed (please see detailed information about the 7 parameters in the supplemental methods).

Statistics. Data are presented as means \pm standard deviations. We used unpaired, 2-tailed Student's $t$ tests for comparisons between 2 groups, and 1-way ANOVA with Bonferroni's post hoc test for multiple comparisons. For all experiments, $P<0.05$ was considered to be significant $\left({ }^{*} P<0.05,{ }^{* *} P<0.01\right)$. All inclusion/exclusion criteria were preestablished, and no samples or animals were excluded from the analysis. No statistical method was used to predetermine the sample size. The experiments were randomized, and the investigators were blinded to allocation during experiments and outcome assessment. Specifically, each animal was assigned an identification number using the animal's litter number in combination with the ear tag number. The investigators who conducted experiments (e.g., ACLT/DMM surgery, siRNA injections, ALN injections) were blinded to animal genotypes. Outcome assessments (e.g., OARSI grading) were conducted by 2 independent graders who were not involved directly in the experiments, and outcomes were recorded in the order of animal identification number.

Study approval. Human OA cartilage was obtained from patients undergoing total knee replacement surgery in the Department of Orthopaedic Surgery at Xiangya Hospital. Normal (control) cartilage was obtained postmortem from human subjects with no history of OA. The patients' consent, as well as approval of the local ethics committees, were obtained before harvesting human tissue samples. We maintained all animals in the animal facility of Johns Hopkins University School of Medicine. The experimental protocol was reviewed and approved by the Institutional Animal Care and Use Committee of Johns Hopkins University. 


\section{Author contributions}

SZ and JZ conceived the ideas for experimental designs, conducted most of the experiments, and prepared the manuscript. GZ, MW, and RLS provided some ideas and helped with behavior analysis. SA, YL, and BG helped with histology sections and animal surgery. QZ, ZC, and YY helped with in vivo DRG imaging. YC, TW, MY, MG, SN, LW, and CW helped with immunostaining and human sample histology. JK and DF provided some of the human OA samples. HKE provided Netrin1-floxed mice. YG helped with behavioral tests and helped write the manuscript. JC helped with manuscript revisions. YH, HWO, XD, and FQZ provided suggestions for the project. $\mathrm{XC}$ developed the concept, supervised the project, conceived the experiments, and wrote most of the manuscript.

\section{Acknowledgments}

This study was supported by NIH National Institute of Arthritis and Musculoskeletal and Skin Diseases grants AR071432 and AR063943 (to XC) and NS070814 (to YG). This work was facilitated by the Pain Research Core funded by the Blaustein Fund and the Neurosurgery Pain Research Institute at Johns Hopkins University, as well as a training award (to SZ) from the China Scholarship Council. The authors thank Jenni Weems and Rachel Box in the editorial office at the Department of Orthopaedic Surgery, Johns Hopkins University, for editing the manuscript.

Address correspondence to: $\mathrm{Xu}$ Cao, 601 North Caroline Street, Suite 5214 Baltimore, Maryland 21287-0881, USA. Phone: 410.502.6440; Email: xcao11@jhmi.edu.
1. Hootman JM, Helmick CG, Barbour KE, Theis KA, Boring MA. Updated projected prevalence of self-reported doctor-diagnosed arthritis and arthritis-attributable activity limitation among US adults, 2015-2040. Arthritis Rheumatol. 2016;68(7):1582-1587.

2. Hunter DJ, McDougall JJ, Keefe FJ. The symptoms of osteoarthritis and the genesis of pain. Med Clin North Am. 2009;93(1):83-100, xi.

3. O'Neil CK, Hanlon JT, Marcum ZA. Adverse effects of analgesics commonly used by older adults with osteoarthritis: focus on non-opioid and opioid analgesics. Am J Geriatr Pharmacother. 2012;10(6):331-342.

4. Lane NE, et al. Tanezumab for the treatment of pain from osteoarthritis of the knee. N Engl JMed. 2010;363(16):1521-1531.

5. Mullard A. Drug developers reboot anti-NGF pain programmes. Nat Rev Drug Discov. 2015;14(5):297-298.

6. Schaible HG, et al. Joint pain. Exp Brain Res. 2009;196(1):153-162.

7. Suokas AK, et al. Quantitative sensory testing in painful osteoarthritis: a systematic review and meta-analysis. Osteoarthr Cartil. 2012;20(10):1075-1085.

8. Hassan H, Walsh DA. Central pain processing in osteoarthritis: implications for treatment. Pain Manag. 2014;4(1):45-56.

9. Lluch E, Torres R, Nijs J, Van Oosterwijck J. Evidence for central sensitization in patients with osteoarthritis pain: a systematic literature review. Eur JPain. 2014;18(10):1367-1375.

10. Miller RE, et al. CCR2 chemokine receptor signaling mediates pain in experimental osteoarthritis. Proc Natl Acad Sci U S A. 2012;109(50):20602-20607.

11. Segond von Banchet G, Boettger MK, Fischer N, Gajda M, Bräuer R, Schaible HG. Experimental arthritis causes tumor necrosis factor-alphadependent infiltration of macrophages into rat dorsal root ganglia which correlates with painrelated behavior. Pain. 2009;145(1-2):151-159.

12. Jin X, Gereau RW. Acute p38-mediated modulation of tetrodotoxin-resistant sodium channels in mouse sensory neurons by tumor necrosis factor-alpha. J Neurosci. 2006;26(1):246-255.

13. Ebbinghaus $M$, et al. The role of interleukin-1 $\beta$ in arthritic pain: main involvement in thermal, but not mechanical, hyperalgesia in rat antigen-induced arthritis. Arthritis Rheum. 2012;64(12):3897-3907.

14. von Banchet GS, Fischer N, Uhlig B, Hensellek S, Eitner A, Schaible HG. Molecular effects of interleukin-1 $\beta$ on dorsal root ganglion neurons: prevention of ligand-induced internalization of the bradykinin 2 receptor and downregulation of $\mathrm{G}$ protein-coupled receptor kinase 2. Mol Cell Neurosci. 2011;46(1):262-271.

15. Obreja O, et al. Fast modulation of heatactivated ionic current by proinflammatory interleukin 6 in rat sensory neurons. Brain. 2005;128(Pt 7):1634-1641.

16. von Banchet GS, Kiehl M, Schaible HG. Acute and long-term effects of IL- 6 on cultured dorsal root ganglion neurones from adult rat. J Neurochem. 2005;94(1):238-248.

17. Richter F, et al. Interleukin-17 sensitizes joint nociceptors to mechanical stimuli and contributes to arthritic pain through neuronal interleukin-17 receptors in rodents. Arthritis Rheum. 2012;64(12):4125-4134.

18. Segond von Banchet G, Boettger MK, König C, Iwakura Y, Bräuer R, Schaible HG. Neuronal IL-17 receptor upregulates TRPV4 but not TRPV1 receptors in DRG neurons and mediates mechanical but not thermal hyperalgesia. Mol Cell Neurosci. 2013;52:152-160.

19. Ashraf S, Mapp PI, Burston J, Bennett AJ, Chapman V, Walsh DA. Augmented pain behavioural responses to intra-articular injection of nerve growth factor in two animal models of osteoarthritis. Ann Rheum Dis. 2014;73(9):1710-1718.

20. Nwosu LN, Mapp PI, Chapman V, Walsh DA. Blocking the tropomyosin receptor kinase $\mathrm{A}$ (TrkA) receptor inhibits pain behaviour in two rat models of osteoarthritis. Ann Rheum Dis. 2016;75(6):1246-1254.

21. Sagar DR, Nwosu L, Walsh DA, Chapman V. Dissecting the contribution of knee joint NGF to spinal nociceptive sensitization in a model of OA pain in the rat. Osteoarthr Cartil. 2015;23(6):906-913.

22. Woolf CJ, Safieh-Garabedian B, Ma QP, Crilly P, Winter J. Nerve growth factor contributes to the generation of inflammatory sensory hypersensitivity. Neuroscience. 1994;62(2):327-331.

23. Miller RJ, Jung H, Bhangoo SK, White FA.
Cytokine and chemokine regulation of sensory neuron function. Handb Exp Pharmacol. 2009;(194):417-449.

24. Vane JR. The mode of action of aspirin-like drugs. Agents Actions. 1978;8(4):430-431.

25. Syx D, Tran PB, Miller RE, Malfait AM. Peripheral mechanisms contributing to osteoarthritis pain. Curr Rheumatol Rep. 2018;20(2):9.

26. Kc R, et al. PKC $\delta$ null mutations in a mouse model of osteoarthritis alter osteoarthritic pain independently of joint pathology by augmenting NGF/TrkA-induced axonal outgrowth. Ann Rheum Dis. 2016;75(12):2133-2141.

27. Ikeuchi M, Wang Q, Izumi M, Tani T. Nociceptive sensory innervation of the posterior cruciate ligament in osteoarthritic knees. Arch Orthop Trauma Surg. 2012;132(6):891-895.

28. Mapp PI, Walsh DA. Mechanisms and targets of angiogenesis and nerve growth in osteoarthritis. Nat Rev Rheumatol. 2012;8(7):390-398.

29. Suri S, Gill SE, Massena de Camin S, Wilson D, McWilliams DF, Walsh DA. Neurovascular invasion at the osteochondral junction and in osteophytes in osteoarthritis. Ann Rheum Dis. 2007;66(11):1423-1428.

30. Malfait AM, Schnitzer TJ. Towards a mechanism-based approach to pain management in osteoarthritis. Nat Rev Rheumatol. 2013;9(11):654-664.

31. Murakami K, Nakagawa H, Nishimura K, Matsuo S. Changes in peptidergic fiber density in the synovium of mice with collagenaseinduced acute arthritis. Can J Physiol Pharmacol. 2015;93(6):435-441.

32. Buma P, Verschuren C, Versleyen D, Van der Kraan P, Oestreicher AB. Calcitonin gene-related peptide, substance $P$ and GAP-43/B-50 immunoreactivity in the normal and arthrotic knee joint of the mouse. Histochemistry. 1992;98(5):327-339.

33. Franchi A, Zaccherotti G, Aglietti P. Neural system of the human posterior cruciate ligament in osteoarthritis. J Arthroplasty. 1995;10(5):679-682.

34. Kwoh CK. Clinical relevance of bone marrow lesions in OA. Nat Rev Rheumatol. 2013;9(1):7-8.

35. Yusuf E, Kortekaas MC, Watt I, Huizinga TW, Kloppenburg M. Do knee abnormalities visualised on MRI explain knee pain in knee osteoarthritis? A systematic review. Ann Rheum Dis. 2011;70(1):60-67. 
36. Laslett LL, et al. Zoledronic acid reduces knee pain and bone marrow lesions over 1 year: a randomised controlled trial. Ann Rheum Dis. 2012;71(8):1322-1328.

37. Laslett LL, Kingsbury SR, Hensor EM, Bowes MA, Conaghan PG. Effect of bisphosphonate use in patients with symptomatic and radiographic knee osteoarthritis: data from the Osteoarthritis Initiative. Ann Rheum Dis. 2014;73(5):824-830.

38. Zhen G, Cao X. Targeting TGF $\beta$ signaling in subchondral bone and articular cartilage homeostasis. Trends Pharmacol Sci. 2014;35(5):227-236.

39. Zhen $\mathrm{G}$, et al. Inhibition of TGF- $\beta$ signaling in mesenchymal stem cells of subchondral bone attenuates osteoarthritis. Nat Med. 2013;19(6):704-712.

40. Xie L, et al. Systemic neutralization of TGF- $\beta$ attenuates osteoarthritis. Ann N Y Acad Sci. 2016;1376(1):53-64.

41. Raper J, Mason C. Cellular strategies of axonal pathfinding. Cold Spring Harb Perspect Biol. 2010;2(9):a001933.

42. Chacón MR, Fazzari P. FAK: dynamic integration of guidance signals at the growth cone. Cell Adh Migr. 2011;5(1):52-55.

43. Moore SW, Zhang X, Lynch CD, Sheetz MP. Netrin-1 attracts axons through FAK-dependent mechanotransduction. JNeurosci. 2012;32(34):11574-11585.

44. Delorme G, Saltel F, Bonnelye E, Jurdic P, Machuca-Gayet I. Expression and function of semaphorin 7A in bone cells. Biol Cell. 2005;97(7):589-597.

45. Fukuda T, et al. Sema3A regulates bone-mass accrual through sensory innervations. Nature. 2013;497(7450):490-493.

46. Irie $\mathrm{N}$, et al. Bidirectional signaling through ephrinA2-EphA2 enhances osteoclastogenesis and suppresses osteoblastogenesis. J Biol Chem. 2009;284(21):14637-14644.

47. Maruyama K, et al. Bone-protective functions of Netrin 1 protein. J Biol Chem. 2016;291(46):23854-23868.

48. Mediero A, Ramkhelawon B, Perez-Aso M, Moore KJ, Cronstein BN. Netrin-1 is a critical autocrine/paracrine factor for osteoclast differentiation. J Bone Miner Res. 2015;30(5):837-854.

49. Sutton AL, Zhang X, Dowd DR, Kharode YP, Komm BS, Macdonald PN. Semaphorin $3 \mathrm{~B}$ is a 1,25-dihydroxyvitamin D3-induced gene in osteoblasts that promotes osteoclastogenesis and induces osteopenia in mice. Mol Endocrinol. 2008;22(6):1370-1381.

50. Takegahara N, et al. Plexin-A1 and its interaction with DAP12 in immune responses and bone homeostasis. Nat Cell Biol. 2006;8(6):615-622.

51. Zhao C, et al. Bidirectional ephrinB2-EphB4 signaling controls bone homeostasis. Cell Metab. 2006;4(2):111-121.

52. Xie H, et al. PDGF-BB secreted by preosteoclasts induces angiogenesis during coupling with osteogenesis. Nat Med. 2014;20(11):1270-1278.

53. Usoskin D, et al. Unbiased classification of sensory neuron types by large-scale single-cell RNA sequencing. Nat Neurosci. 2015;18(1):145-153.

54. Glasson SS, Blanchet TJ, Morris EA. The surgical destabilization of the medial meniscus (DMM) model of osteoarthritis in the 129/SvEv mouse.
Osteoarthr Cartil. 2007;15(9):1061-1069.

55. Kim AY, et al. Pirt, a phosphoinositide-binding protein, functions as a regulatory subunit of TRPV1. Cell. 2008;133(3):475-485.

56. Kim YS, et al. Central terminal sensitization of TRPV1 by descending serotonergic facilitation modulates chronic pain. Neuron . 2014;81(4):873-887.

57. Miller RE, et al. Visualization of peripheral neuron sensitization in a surgical mouse model of osteoarthritis by in vivo calcium imaging. Arthritis Rheumatol. 2018;70(1):88-97.

58. Nakashima T, et al. Evidence for osteocyte regulation of bone homeostasis through RANKL expression. Nat Med. 2011;17(10):1231-1234.

59. Xiong J, Onal M, Jilka RL, Weinstein RS, Manolagas SC, O’Brien CA. Matrix-embedded cells control osteoclast formation. Nat Med. 2011;17(10):1235-1241.

60. Tang Y, et al. TGF-beta1-induced migration of bone mesenchymal stem cells couples bone resorption with formation. Nat Med. 2009;15(7):757-765

61. Chaplan SR, Bach FW, Pogrel JW, Chung JM, Yaksh TL. Quantitative assessment of tactile allodynia in the rat paw. JNeurosci Methods. 1994;53(1):55-63.

62. Taylor AM, Blurton-Jones M, Rhee SW, Cribbs DH, Cotman CW, Jeon NL. A microfluidic culture platform for CNS axonal injury, regeneration and transport. Nat Methods. 2005;2(8):599-605

63. Bashaw GJ, Klein R. Signaling from axon guidance receptors. Cold Spring Harb Perspect Biol. 2010;2(5):a001941.

64. Chen JS, Sambrook PN. Antiresorptive therapies for osteoporosis: a clinical overview. Nat Rev Endocrinol. 2011;8(2):81-91.

65. Ding M, Danielsen CC, Hvid I. The effects of bone remodeling inhibition by alendronate on three-dimensional microarchitecture of subchondral bone tissues in guinea pig primary osteoarthrosis. Calcif Tissue Int. 2008;82(1):77-86.

66. Hayami T, et al. The role of subchondral bone remodeling in osteoarthritis: reduction of cartilage degeneration and prevention of osteophyte formation by alendronate in the rat anterior cruciate ligament transection model. Arthritis Rheum. 2004;50(4):1193-1206.

67. Jones MD, Tran CW, Li G, Maksymowych WP, Zernicke RF, Doschak MR. In vivo microfocal computed tomography and micro-magnetic resonance imaging evaluation of antiresorptive and antiinflammatory drugs as preventive treatments of osteoarthritis in the rat. Arthritis Rheum. 2010;62(9):2726-2735.

68. Fang H, Beier F. Mouse models of osteoarthritis: modelling risk factors and assessing outcomes. Nat Rev Rheumatol. 2014;10(7):413-421.

69. Hochberg MC, et al. American College of Rheumatology 2012 recommendations for the use of nonpharmacologic and pharmacologic therapies in osteoarthritis of the hand, hip, and knee. Arthritis Care Res (Hoboken). 2012;64(4):465-474.

70. Cui Z, et al. Halofuginone attenuates osteoarthritis by inhibition of TGF- $\beta$ activity and H-type vessel formation in subchondral bone. Ann Rheum Dis. 2016;75(9):1714-1721.

71. Sharma AR, Jagga S, Lee SS, Nam JS. Interplay between cartilage and subchondral bone contributing to pathogenesis of osteoarthritis. Int $\mathrm{J} \mathrm{Mol}$ Sci. 2013;14(10):19805-19830.

72. Miller RE, et al. Chemogenetic inhibition of pain neurons in a mouse model of osteoarthritis. Arthritis Rheumatol. 2017;69(7):1429-1439.

73. Vogel G. Developmental biology. The unexpected brains behind blood vessel growth. Science. 2005;307(5710):665-667.

74. Layne K, Ferro A, Passacquale G. Netrin-1 as a novel therapeutic target in cardiovascular disease: to activate or inhibit? Cardiovasc Res. 2015;107(4):410-419.

75. Park KW, et al. The axonal attractant Netrin- 1 is an angiogenic factor. Proc Natl Acad Sci US A. 2004;101(46):16210-16215.

76. Wilson BD, et al. Netrins promote developmental and therapeutic angiogenesis. Science. 2006;313(5787):640-644.

77. Tu T, et al. CD146 acts as a novel receptor for netrin-1 in promoting angiogenesis and vascular development. Cell Res. 2015;25(3):275-287.

78. Mehlen P, Furne C. Netrin-1: when a neuronal guidance cue turns out to be a regulator of tumorigenesis. Cell Mol Life Sci. 2005;62(22):2599-2616.

79. Yoneda T, Hiasa M, Nagata Y, Okui T, White FA. Acidic microenvironment and bone pain in cancercolonized bone. Bonekey Rep. 2015;4:690.

80. Wu CH, et al. Netrin-1 contributes to myelinated afferent fiber sprouting and neuropathic pain. Mol Neurobiol. 2016;53(8):5640-5651.

81. Snider WD, McMahon SB. Tackling pain at the source: new ideas about nociceptors. Neuron. 1998;20(4):629-632.

82. Dong X, Han S, Zylka MJ, Simon MI, Anderson DJ. A diverse family of GPCRs expressed in specific subsets of nociceptive sensory neurons. Cell. 2001;106(5):619-632.

83. Genç B, et al. Visualization of sensory neurons and their projections in an upper motor neuron reporter line. PLoS ONE. 2015;10(7):e0132815.

84. Aso K, Izumi M, Sugimura N, Okanoue Y, Ushida T, Ikeuchi M. Nociceptive phenotype alterations of dorsal root ganglia neurons innervating the subchondral bone in osteoarthritic rat knee joints. Osteoarthr Cartil. 2016;24(9):1596-1603.

85. Varma AK. Charcot neuroarthropathy of the foot and ankle: a review. J Foot Ankle Surg. 2013;52(6):740-749.

86. Larson SA, Burns PR. The pathogenesis of Charcot neuroarthropathy: current concepts. Diabet Foot Ankle. 2012;3.

87. Corset V, Nguyen-Ba-Charvet KT, Forcet C, Moyse E, Chédotal A, Mehlen P. Netrin-1mediated axon outgrowth and cAMP production requires interaction with adenosine $\mathrm{A} 2 \mathrm{~b}$ receptor. Nature. 2000;407(6805):747-750.

88. Tak E, et al. Protective role for netrin-1 during diabetic nephropathy. J Mol Med. 2013;91(9):1071-1080.

89. Rosenberger P, et al. Hypoxia-inducible factordependent induction of netrin-1 dampens inflammation caused by hypoxia. Nat Immunol. 2009;10(2):195-202.

90. Abo-Salem OM, Hayallah AM, Bilkei-Gorzo A, Filipek B, Zimmer A, Müller CE. Antinociceptive effects of novel A2B adenosine 
receptor antagonists. J Pharmacol Exp Ther. 2004;308(1):358-366.

91. Sawynok J, Yaksh TL. Caffeine as an analgesic adjuvant: a review of pharmacology and mechanisms of action. Pharmacol Rev. 1993;45(1):43-85.

92. Bingham $\mathrm{CO}$, et al. Risedronate decreases biochemical markers of cartilage degradation but does not decrease symptoms or slow radiographic progression in patients with medial compartment osteoarthritis of the knee: results of the two-year multinational knee osteoarthritis structural arthritis study. Arthritis Rheum. 2006;54(11):3494-3507.

93. Buckland-Wright JC, Messent EA, Bingham CO,
Ward RJ, Tonkin C. A 2 yr longitudinal radiographic study examining the effect of a bisphosphonate (risedronate) upon subchondral bone loss in osteoarthritic knee patients. Rheumatology (Oxford). 2007;46(2):257-264.

94. Raisz L, et al. Short-term risedronate treatment in postmenopausal women: effects on biochemical markers of bone turnover. Osteoporos Int 2000;11(7):615-620.

95. Spector TD, et al. Effect of risedronate on joint structure and symptoms of knee osteoarthritis: results of the BRISK randomized, controlled trial [ISRCTN01928173]. Arthritis Res Ther. 2005;7(3):R625-R633.
96. Castañeda S, Roman-Blas JA, Largo R, Herrero-Beaumont G. Subchondral bone as a key target for osteoarthritis treatment. Biochem Pharmacol.2012;83(3):315-323.

97. Solomon LA, Russell BA, Watson LA, Beier F, Bérubé NG. Targeted loss of the ATR-X syndrome protein in the limb mesenchyme of mice causes brachydactyly. Hum Mol Genet. 2013;22(24):5015-5025.

98. Hamers FP, Lankhorst AJ, van Laar TJ, Veldhuis WB, Gispen WH. Automated quantitative gait analysis during overground locomotion in the rat: its application to spinal cord contusion and transection injuries. J Neurotrauma. 2001;18(2):187-201. 